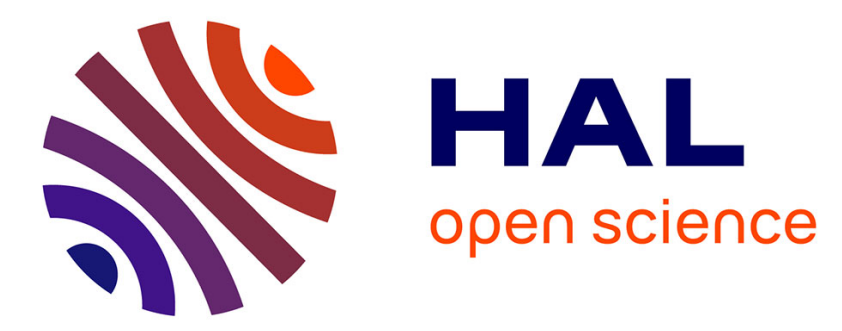

\title{
Flow regimes in a vertical Taylor-Couette system with a radial thermal gradient
}

\author{
Raphaël Guillerm, Changwoo Kang, C Savaro, Valérie Lepiller, Arnaud \\ Prigent, K Yang, Innocent Mutabazi
}

\section{- To cite this version:}

Raphaël Guillerm, Changwoo Kang, C Savaro, Valérie Lepiller, Arnaud Prigent, et al.. Flow regimes in a vertical Taylor-Couette system with a radial thermal gradient. Physics of Fluids, 2015, 27 (9), pp.094101. 10.1063/1.4930588 . hal-02868251

\section{HAL Id: hal-02868251 \\ https://hal.science/hal-02868251}

Submitted on 15 Jun 2020

HAL is a multi-disciplinary open access archive for the deposit and dissemination of scientific research documents, whether they are published or not. The documents may come from teaching and research institutions in France or abroad, or from public or private research centers.
L'archive ouverte pluridisciplinaire HAL, est destinée au dépôt et à la diffusion de documents scientifiques de niveau recherche, publiés ou non, émanant des établissements d'enseignement et de recherche français ou étrangers, des laboratoires publics ou privés. 


\title{
Flow regimes in a vertical Taylor-Couette system with a radial thermal gradient
}

\author{
R. Guillerm ${ }^{1}$, C. Kang ${ }^{2}$, C. Savaro ${ }^{1}$, V. Lepiller ${ }^{1 \bullet}$, A. Prigent ${ }^{1}$, K.-S. Yang ${ }^{2}$ and I.Mutabazi $^{1 *}$ \\ ${ }^{1}$ Laboratoire Ondes et Milieux Complexes (LOMC), UMR 6294, CNRS-Université du Havre, F-76058 \\ Le Havre Cedex, France
}

${ }^{2}$ Department of Mechanical Engineering, INHA University, Incheon 402-751, Republic of Korea

\begin{abstract}
$\underline{\text { Abstract }}$
A rich variety of flow regimes in a Newtonian fluid inside a vertical large-aspect ratio and a wide-gap Taylor-Couette system with a radial temperature gradient has been determined in experiments and in direct numerical simulations (DNS). Compared to previous experiments and numerical studies, a wider range of temperature differences (i.e. of the Grashof number $\mathrm{Gr}$ ) and of the rotation rate (the Taylor number $\mathrm{Ta}$ ) has been covered. The combined effect of rotation and of the radial temperature gradient is the occurrence of helicoidal vortices or modulated waves at the onset. Stationary axisymmetric vortices are found for very weak temperature differences. A good agreement was found for critical states between results from experiments, linear stability analysis and DNS. Higher instability modes have been determined for a wide range of parameters and a state diagram of observable flow regimes has been established in the plane spanned by $G \mathrm{r}$ and $T a$. Some higher states observed in experiments were retrieved in DNS.
\end{abstract}

\section{INTRODUCTION}

Flow in a differentially rotating cylindrical annulus with a radial temperature gradient and a fixed outer cylinder occurs in rotating machineries such as electric motors or generators [1-5]. There are other applications such as high speed pumps used in BWR (Boiling Water Reactors) to transport water at high pressure and temperature [6] or in solidification of pure metals where the variation of heat transport greatly affects the crystal-melt interface $[7,8]$. The flow system may be encountered in more complex processes such as drilling where a rotating rod descends or ascends in an environment with both radial and vertical temperature gradients [9]. The flow in these systems is more complex than the circular Couette flow (CCF) as it results from both the rotation and the temperature gradient. For the isothermal case, the rotation induces CCF which is potentially unstable to centrifugally driven perturbations

\footnotetext{
• Permanent address: Institut FEMTO-ST, UMR 6174, CNRS-Université de Franche Comté, 2, Avenue jean Moulin, F-90000, Belfort Cedex

*Author to whom correspondence should be sent : innocent.mutabazi@univ-lehavre.fr
} 
leading to Taylor vortex flow (TVF) when a critical value of the rotation rate is exceeded [10, 11]. The radial temperature gradient induces a baroclinic flow which, for small values of the temperature gradient, consists of one convection cell (ascending near the hot surface and descending near the cold one). For large values, this baroclinic flow becomes unstable leading to convective cells inside the large convection cell [12-15]. Therefore, the combination of a cylinder rotation and a radial temperature gradient leads to a complex flow with a rich variety of the flow regimes when the control parameters are increased. In particular, critical states are in most cases time-dependent with temperature fluctuations of low frequencies. The latter can induce thermal stresses which may lead to material fatigue after long term use [6]. The problem contains a large number of parameters, the most important being the temperature difference and the rotation rate, the other ones being fixed or weakly varying in most of practical situations.

Despite its multiple applications, this flow system has been investigated experimentally and numerically only by few authors [16-21]. Snyder and Karlsson [16] in a pioneering experimental work in water flow gave the diagram of critical states which shows that the threshold increases for small temperature differences (i.e. the CCF is stabilized) while it decreases for large temperature differences and the critical modes form a spiral vortex flow or spiral pattern (SPI) of uneven size. Sorour and Coney [17] have used high viscous oil and found only destabilization of the base flow to SPI. Ball et al. [18] performed experimental investigations of an air flow and classified the critical flow regimes using the Richardson number focusing on the destabilization of the convective cell to spiral vortex. While the studies [16, 17] used large aspect ratio systems, the experiment of Ball et al. [18] was performed in a relatively small aspect ratio system where the end effects had significant influence on the transition parameters. Lepiller et al. [21] performed an experiment in a large aspect-ratio system with water and found that the temperature difference is destabilizing. All these studies concentrated mainly on the first transition from the base flow to the first instability modes. A thorough linear stability analysis has been performed by Ali and Weidman [19] who investigated the instability threshold parameters for small- and wide-gap annulus and for several values of the Prandtl number. Kuo and Ball [20] and Kedia et al. [6] performed numerical simulations for some values of $G r$ and $R e$. Based on results of Lepiller et al. [21,22], Yoshikawa et al. [23] revisited the linear stability for a cylindrical annulus with the same radius ratio as that of [21] and complemented it with an energetic analysis which allowed separation of the centrifugal from the buoyancy effects and the shear-driven instability. Kang et al. [24, 25] have performed the numerical simulations DNS for fixed 
values of $T a$ and increased the Grashof number in order to investigate the flow destabilization by thermal effects. The present study is a continuation of these studies and is concerned with experimental and numerical simulations of the CCF when the driving forces are increased in magnitude. The experiment covers a larger range of temperature differences (leading to $\mathrm{Gr} \in$ $\left.\left[0 ; 10^{4}\right]\right)$ than that covered in previous studies. The experiment and the DNS are performed for a fixed value of the temperature difference and a continuous increase in the rotation rate.

The paper is organized as follows. The next section recalls the flow equations that govern the system together with the equations of the variation of the kinetic energy and of the momentum and heat transfer. In section 3, numerical results are presented. The setup and the experimental results are described in section 4 . The last section addresses the discussion and conclusion.

\section{PROBLEM FORMULATION}

\section{A. Flow equations and control parameters}

We investigate the stability of an incompressible Newtonian fluid confined in a cylindrical annulus with the inner cylinder rotating at the angular frequency $\Omega$ and an imposed radial temperature difference $\delta T$ in the gravitational field $\vec{g}=-g \vec{e}_{z}$. The inner cylinder has a radius $a$ and the outer cylinder radius is $b$. The cylindrical annulus has a length $H$ and a width $d=b-a$ (Fig.1). The fluid has a density $\rho$, a kinematic viscosity $v$, a thermal diffusivity $\kappa$ and a thermal expansion coefficient $\alpha$. Because of different driving forces, it is useful to introduce the dimensionless parameters using the ratios of timescales. The viscous diffusion timescale $\tau_{v}=d^{2} / v$, the thermal diffusion timescale $\tau_{\kappa}=d^{2} / \kappa$, the Archimedean timescale $\tau_{A}=\sqrt{d / \alpha \delta T g}$ and the centrifugal timescale $\tau_{c}=\sqrt{d / \Omega^{2} a}$. So we define the Prandtl number $\operatorname{Pr}=\tau_{\kappa} / \tau_{v}=v / \kappa$, the Grashof number $\operatorname{Gr}=\left(\tau_{v} / \tau_{A}\right)^{2}=\alpha \delta \operatorname{Tgd}^{3} / v^{2}$ and the Taylor number $T a=\tau_{v} / \tau_{c}=\operatorname{Re} \sqrt{d / a}$ with the Reynolds number $\operatorname{Re}=\Omega a d / v$. The Galileo number $G a=g d^{3} / v^{2}$ defines the flow system together with the geometrical parameters $\Gamma=H / d$ and $\eta=a / b$. The Richardson number $R i=G r / \operatorname{Re}^{2}$ gives the relative importance of the Archimedean buoyancy to the centrifugal force.

The governing flow equations are the laws of conservation of mass, linear momentum and energy. We assume that the temperature difference imposed on the cylinders is small so that the Boussinesq approximation is valid. This allows us to neglect the density variation in 
all terms except when the density is multiplied by the gravity $g$ and the centrifugal acceleration. The Boussinesq equations are made dimensionless using the gap width $d$ as a length scale, $\mathrm{d} / \Omega a$ as a timescale and $\delta T$ as a temperature scale. In the cylindrical coordinates $(r, \varphi, z)$, they read:

$$
\begin{aligned}
& \frac{1}{r} \frac{\partial}{\partial r}\left(r u_{r}\right)+\frac{1}{r} \frac{\partial u_{\varphi}}{\partial \varphi}+\frac{\partial u_{z}}{\partial z}=0 \\
& \frac{\partial u_{r}}{\partial t}+(\vec{u} \cdot \vec{\nabla}) u_{r}-\left[1-\gamma_{\alpha} T\right] \frac{u_{\varphi}^{2}}{r}=-\frac{\partial P}{\partial r}+\frac{1}{\operatorname{Re}}\left[\left(\nabla^{2}-\frac{1}{r^{2}}\right) u_{r}-\frac{2}{r^{2}} \frac{\partial u_{\varphi}}{\partial \varphi}\right] \\
& \frac{\partial u_{\varphi}}{\partial t}+(\vec{u} \cdot \vec{\nabla}) u_{\varphi}+\frac{u_{r} u_{\varphi}}{r}=-\frac{\partial p}{r \partial \varphi}+\frac{1}{\operatorname{Re}}\left[\left(\nabla^{2}-\frac{1}{r^{2}}\right) u_{\varphi}+\frac{2}{r^{2}} \frac{\partial u_{r}}{\partial \varphi}\right] \\
& \frac{\partial u_{z}}{\partial t}+(\vec{u} \cdot \vec{\nabla}) u_{z}=-\frac{\partial P}{\partial z}+\frac{1}{\operatorname{Re}} \nabla^{2} u_{z}+\operatorname{RiT} \\
& \frac{\partial T}{\partial t}+(\vec{u} \cdot \vec{\nabla}) T=\frac{1}{\operatorname{Pr} \operatorname{Re}} \nabla^{2} T .
\end{aligned}
$$

where $u_{r}, u_{\varphi}, u_{z}$ denote the radial, azimuthal and axial velocity components respectively, $P$ is the pressure and $T$ is the temperature. We have also introduced the operators

$\vec{u} \cdot \vec{\nabla} \equiv u_{r} \frac{\partial}{\partial r}+\frac{u_{\varphi}}{r} \frac{\partial}{\partial \varphi}+u_{z} \frac{\partial}{\partial z}$ and $\nabla^{2} \equiv \frac{1}{r} \frac{\partial}{\partial r}\left(r \frac{\partial}{\partial r}\right)+\frac{1}{r^{2}} \frac{\partial^{2}}{\partial \varphi^{2}}+\frac{\partial^{2}}{\partial z^{2}}$.

The equations (1-b) and (1-d) contain the main driving forces of instability in the flow, i.e. the centrifugal force $u_{\varphi}^{2} / r$, the centrifugal buoyancy $\gamma_{\alpha} T u_{\varphi}^{2} / r$ with $\gamma_{\alpha}=\alpha \delta T$ and the Archimedean buoyancy RiT .

The boundary conditions are the no-slip on the cylindrical surfaces including the endplates with, isothermal conditions on lateral surfaces and adiabatic conditions on top and bottom endplates:

$$
\left\{\begin{array}{l}
u_{r}=0, \quad u_{\varphi}=\operatorname{Re}, u_{z}=0 ; T=1 \quad \text { at } r=\bar{a}=\eta /(1-\eta) \\
u_{r}=0, \quad u_{\varphi}=0, \quad u_{z}=0 ; T=0 \quad \text { at } r=\bar{b}=1 /(1-\eta) \\
u_{r}=0, \quad u_{\varphi}=0, \quad u_{z}=0 ; \partial T / \partial z=0 \quad \text { at } z=0 \\
u_{r}=0, \quad u_{\varphi}=0, \quad u_{z}=0 ; \partial T / \partial z=0 \quad \text { at } z=\Gamma
\end{array}\right.
$$

In a given flow system, the geometric parameters are fixed ( $\Gamma=$ const, $\eta=$ const $)$ together with the Galileo number $G a$. Therefore, the flow is controlled by 3 independent parameters: the Taylor number $\mathrm{Ta}$ which indicates the strength of the rotation effects, the Grashof number $\mathrm{Gr}$ which gives the magnitude of the temperature difference and the Prandtl 
number $\operatorname{Pr}$. In the numerical simulations where the Boussinesq approximation is assumed to be valid, the Prandtl number is fixed.

Neglecting the end effects near the top and bottom plates (i.e. in the limit of infinite aspect ratio), the velocity profile of the base flow is $\vec{u}(\vec{r})=V(r) \vec{e}_{\varphi}+W(r) \vec{e}_{z}$ where $V(r)$ is the circular Couette profile and $W(r)$ is the baroclinic axial velocity component. Their explicit expressions can be found in [19, 21, 23]. The CCF is potentially unstable to centrifugallydriven perturbations. The axial profile $W(r)$ has an inflexion point with a maximum of vorticity, so that according to the Rayleigh-Förtföt criterion [11], the flow is potentially unstable to transverse perturbations. Moreover, in the case where the inner cylinder is cooled while the outer is heated, the centrifugal acceleration destabilizes the radial stratification of the fluid density [26]. Lepiller et al. [21] have investigated the first transition experimentally from the base flow state for $G r \in[-850 ; 965]$. One of the purposes of the present work is to extend these results to higher states for large values $G r$ up to $G r=5000$. Direct numerical simulations will make it possible to determine flow structures for chosen values of $G r$ in the range where the Boussinesq approximation is still valid.

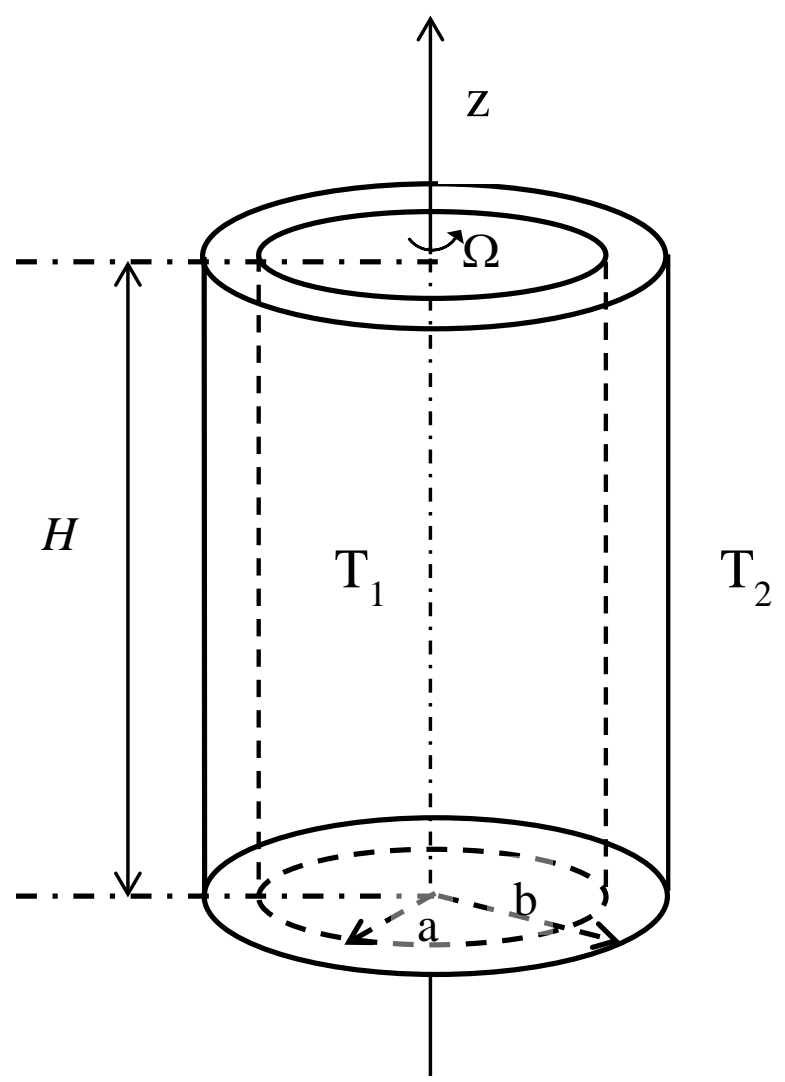

Figure 1: Scheme of the flow configuration: flow inside two coaxial cylinders of radii $a$ and $b$ $=a+d$, and height $H$ maintained at different temperatures $\mathrm{T}_{1}$ and $\mathrm{T}_{2}$. 


\section{B. Kinetic energy, momentum and heat transfer}

The variation rate of the kinetic energy can be obtained using the averaging method developed recently by Eckhardt et al. [27] and it reads:

$$
\operatorname{Re}^{2} \frac{d E_{k}}{d t}=\frac{(1-\eta)^{2}}{\eta(1+\eta)} 2 G+G r\left\langle T u_{z}\right\rangle_{V}+G r \operatorname{Fr}^{2}\left\langle T r u_{r} \omega^{2}\right\rangle_{V}-\operatorname{Re}\langle\varepsilon\rangle
$$

The quantity $G$ is the dimensionless form of the torque $M_{z}$ acting on the inner cylinder, i.e. $G=M_{z} / 2 \pi H \rho v^{2}$. It is related to the dimensionless shear stress $\tau_{r \varphi}$ as follows

$$
G=\left.T a\left(\frac{\eta}{1-\eta}\right)^{5 / 2} \tau_{r \varphi}\right|_{r=\bar{a}}
$$

where the dimensionless stress tensor component is given by

$$
\tau_{r \varphi}(r)=\frac{4}{\operatorname{Re}}\left[r \frac{\partial}{\partial r}\left(\frac{u_{\varphi}}{r}\right)+\frac{1}{r} \frac{\partial u_{r}}{\partial \varphi}\right]
$$

For infinite cylinder annulus with only the inner cylinder rotating, the laminar dimensionless torque is given by

$$
G_{\text {lam }}=\frac{2 T a}{1-\eta^{2}}\left(\frac{\eta}{1-\eta}\right)^{3 / 2}
$$

The first term in the right-hand side (r.h.s.) of (2) represents the power due to rotation, the second term is the power produced by the buoyancy force, the third term is the power due to centrifugal buoyancy. The energy dissipation rate $\varepsilon$ in cylindrical coordinates is given by

$$
\begin{aligned}
\varepsilon= & 2\left[\left(\frac{\partial u_{r}}{\partial r}\right)^{2}+\left(\frac{1}{r} \frac{\partial u_{\varphi}}{\partial \varphi}+\frac{u_{r}}{r}\right)^{2}+\left(\frac{\partial u_{z}}{\partial z}\right)^{2}\right] \\
& +\left[r \frac{\partial}{\partial r}\left(\frac{u_{\varphi}}{r}\right)+\frac{1}{r} \frac{\partial u_{r}}{\partial \varphi}\right]^{2}+\left[\frac{1}{r} \frac{\partial u_{z}}{\partial \varphi}+\frac{\partial u_{\varphi}}{\partial z}\right]^{2}+\left[\frac{\partial u_{r}}{\partial z}+\frac{\partial u_{z}}{\partial r}\right]^{2}
\end{aligned}
$$

The average is taken over the cylindrical surface of area $A=2 \pi r \Gamma$ :

$$
\langle X\rangle_{A}=\int X \frac{r d \varphi d z}{2 \pi r H} \text { with } r \in[\bar{a}, \bar{b}] \text { and } \bar{a}=\eta / 1-\eta, \bar{b}=1 / 1-\eta
$$

In different applications, the measure of the torque $\vec{M}$ acting on the cylinder is the friction coefficient defined as follows [2] :

$$
C_{M_{2}} \equiv \frac{M_{z}}{\pi H \rho \Omega^{2} a^{4} / 2}=\left(\frac{1-\eta}{\eta}\right)^{3} \frac{4 G}{T a^{2}}
$$


The friction coefficient for laminar Couette flow in an isothermal infinite length annulus, is given by

$$
C_{M_{z}}=-\frac{1}{\eta(1+\eta)}\left(\frac{1-\eta}{\eta}\right)^{1 / 2} \frac{8}{T a}
$$

The baroclinic flow induces an azimuthal component of the torque $M_{\varphi}$ which leads to the friction coefficient $C_{M_{\varphi}}$ given by

$$
C_{M_{\varphi}} \equiv \frac{M_{\varphi}}{\pi H \rho \Omega^{2} a^{4} / 2}=\frac{4}{\operatorname{Re}}\left(\frac{\partial u_{z}}{\partial r}+\frac{\partial u_{r}}{\partial z}\right)
$$

The heat transfer in the radial direction is determined by the average of the temperature gradient at the inner cylinder

$$
\bar{N} u_{i}=-\frac{\eta \ln \eta}{1-\eta}\left\langle\left(\frac{\partial T}{\partial r}\right)\right\rangle_{i}
$$

In analogy with thermal convection, Eckhardt et al. [27] have introduced the pseudo-Nusselt number as follows

$$
N_{\omega}=G / G_{l a m}
$$

where $G_{l a m}$ is the dimensionless torque in the laminar flow. The quantity $N_{\omega}$ measures the transfer of the angular velocity in the radial direction and therefore the dissipation rate of the kinetic energy. It is the analogue of the Nusselt number $\mathrm{Nu}$ in thermal convection.

\section{NUMERICAL SIMULATIONS AND RESULTS}

\section{A. Numerical simulations}

We have performed direct numerical simulations of the governing equations which were discretized using a finite-volume method in a cylindrical coordinate system. For the flow field, a second-order accurate central differencing was utilized for spatial discretization. For the temperature field, a central difference scheme was employed for the diffusion terms, and the QUICK (Quadratic Upstream Interpolation for Convective Kinematics) scheme was used for the convective terms. A hybrid scheme was used for time advancement; non-linear terms and cross diffusion terms are explicitly advanced by a third-order Runge-Kutta scheme, and the other terms except for the pressure gradient terms are implicitly advanced by the CrankNicolson scheme. A fractional step method was employed to decouple the continuity and momentum equations. The resulting Poisson equation was solved by a multigrid method. 
Details of the numerical algorithm used in the current code are described in [24, 25, 28]. The grid employed in this study is a body-fitted O-grid system which is the most adequate for the present flow configuration. More resolution is allocated near the cylinder walls where gradients are steep. The number of grid points determined by grid-refinement study was $32 \times 64 \times 1024$ in the radial, azimuthal and axial directions, respectively. In the axial direction, the grid cells are uniform with $\Delta \mathrm{z}=0.11$ which is fine enough to capture the boundary layers near the end plates. The radial coordinate is defined as $x=r-\eta /(1-\eta) \in[0 ; 1]$.

We present the results from both the experiment and the DNS. The DNS have been performed for few specific values of $G r$ because of the computing time cost, due to the choice of long axial domain of aspect ratio $\Gamma=114$, close to the experimental aspect ratio.

\section{B. Numerical results}

In the previous study [24, 25], numerical simulations were performed for the case when Ta was fixed and $\mathrm{Gr}$ was varied in order to investigate the effect of thermal temperature gradient on the momentum transfer and the heat transfer in the annulus. The present study focuses on the flow structures for fixed $\mathrm{Gr}$ and increasing $\mathrm{Ta}$ following the same protocol as in the experiment developed below. For the clarity, we present results obtained for $\mathrm{Gr}=1028$, other numerical data will be used to compare with experimental results in the discussion.

\section{B1. Base flow state}

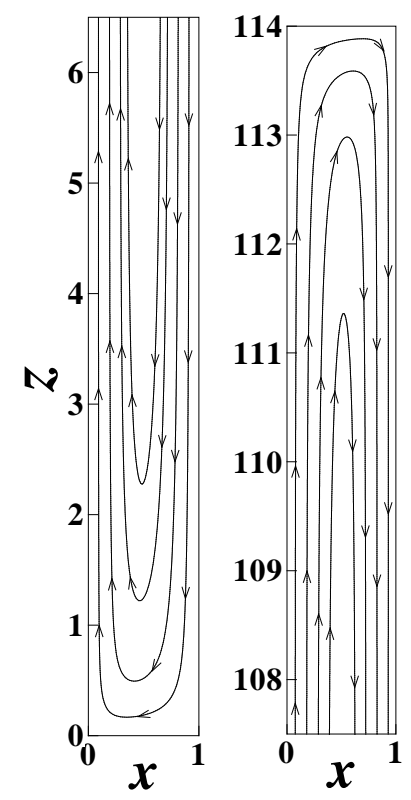

(a)

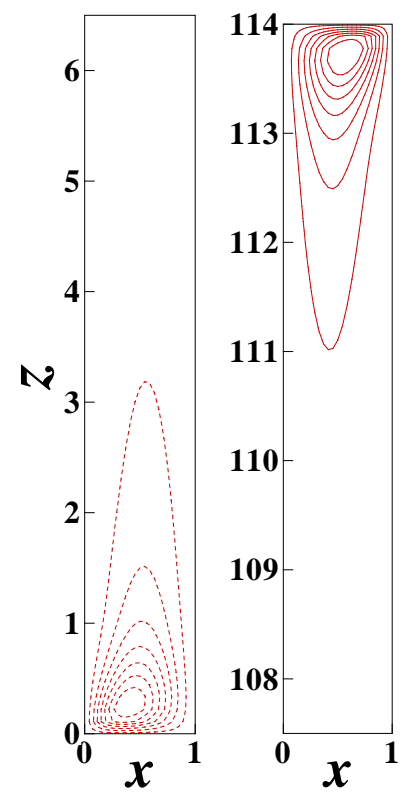

(b)
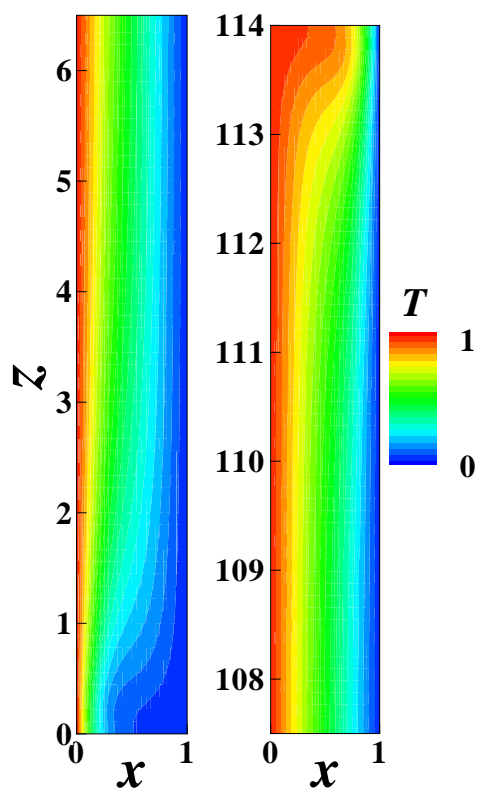

(c)

Figure 2: Base flow and temperature field for $G r=1028, T a=8$ : near the bottom (left), near the top (right) : (a) streamlines, (b) contours of $u_{r}$, (c) contours of temperature. 
In the presence of both temperature difference and rotation of the inner cylinder, the base flow state is two dimensional: it is invariant under rotation but because of the finite length, there is no translational invariance along the axial direction. In the central flow, the flow has two velocity components: the azimuthal component due to the rotation of the inner cylinder and the axial component generated by the radial temperature gradient. Near the endplates, but away from the adiabatic condition at the endplates, there is a vertical temperature gradient (which vanishes away from the plates) and its coupling with the rotation amplifies the formation of the azimuthal vorticity. The flow has three velocity components because of the radial component which ensures the closure of the streamlines. The axial component of the Ekman flow (due to the rotation of the inner cylinder) is amplified by the baroclinic velocity component induced by the radial temperature gradient. The behavior of the velocity and temperature field near the endplates is shown in Figure 2. The radial component $u_{\mathrm{r}}$ and the axial temperature gradient in the base flow are localized near the endplates (Fig. 2 b-c). Our flow system has a large enough aspect ratio such that the end effects will not have a strong influence on the pattern formation.

\section{Flow patterns}

The transition from laminar state leads to a state of corotating vortices (Fig.3) with a well defined wavelength and frequency. Figure 3 shows the flow pattern just above the onset for $G r=1028$ near the endplates and in the central part of the system. At $T a=20$, the pattern is formed of a pattern of corotating vortices. As the rotation increases, the magnitude of the centrifugal force increases and weak-intensity vortices with negative azimuthal vorticity are formed around $T a=45$. Different patterns obtained when increasing $T a$ are represented in Figure 4 and corresponding temperature fields together with the velocity vectors in Figure 5 (only central parts of the patterns are shown). When the centrifugal force becomes very intense, the counter-rotating vortices become stronger and some defects have been observed (Fig.4-c,-d). 


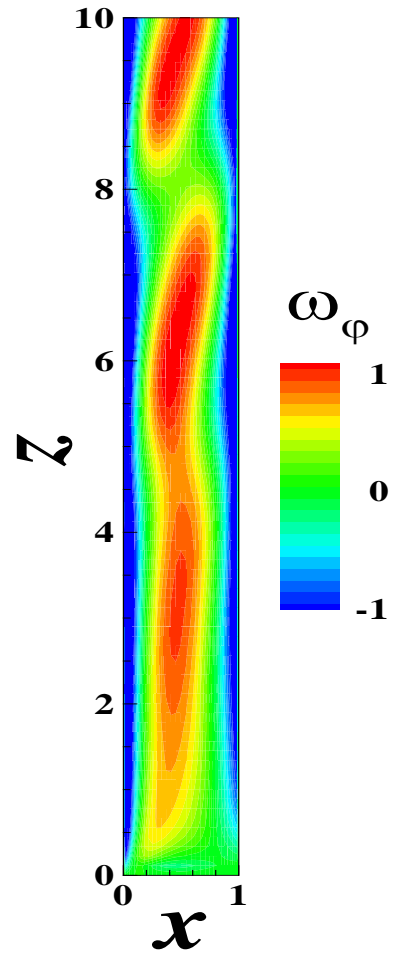

(a)

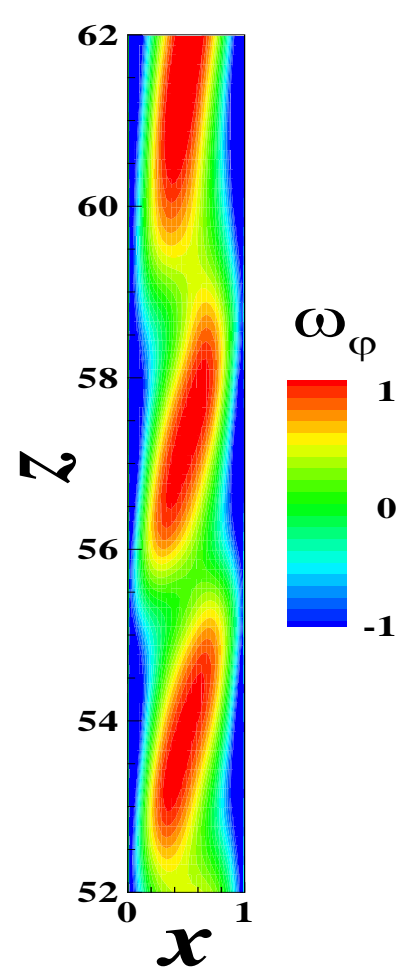

(b)

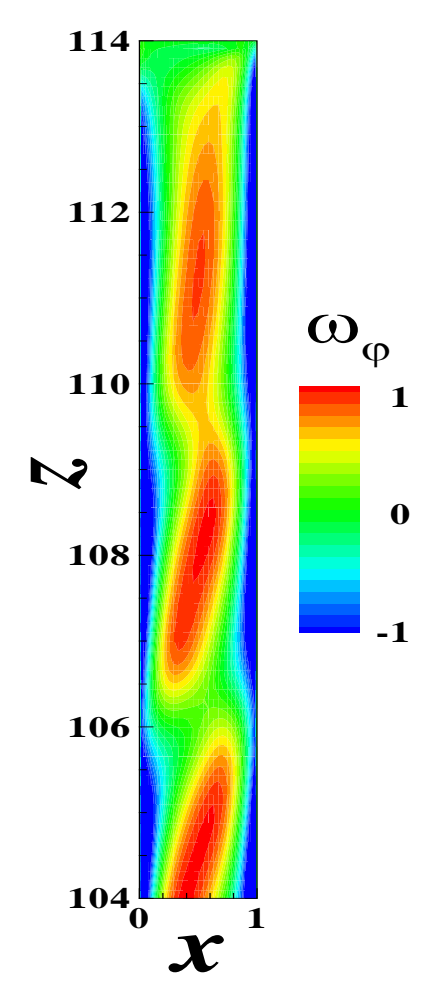

(c)

Figure 3: Contours of azimuthal vorticity to show flow structures for $G r=1028$ for $T a=20:$ a) near the bottom endplate, b) in the central part, c) near the top endplate.

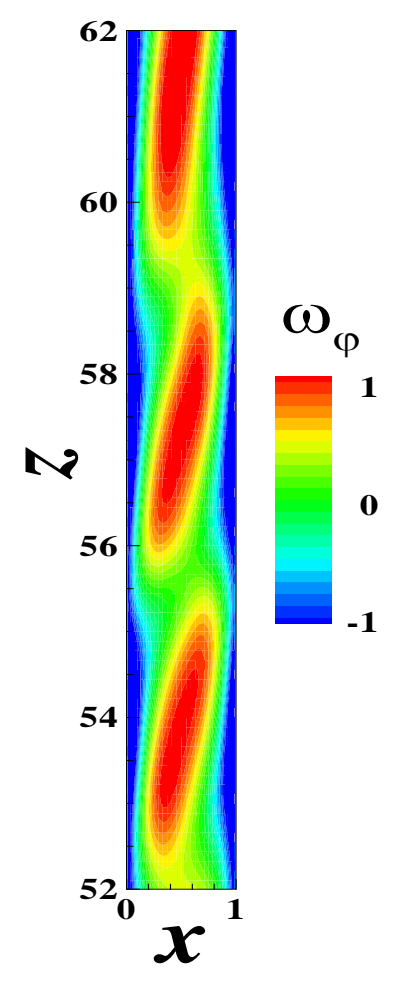

(a)

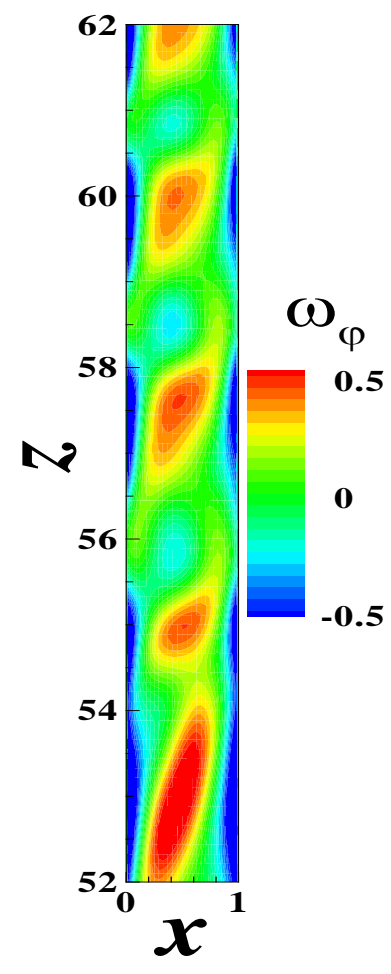

(b)

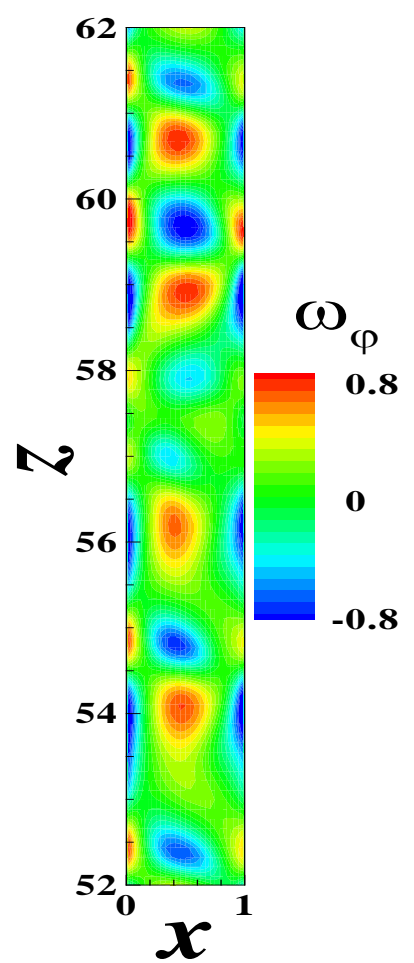

(c)

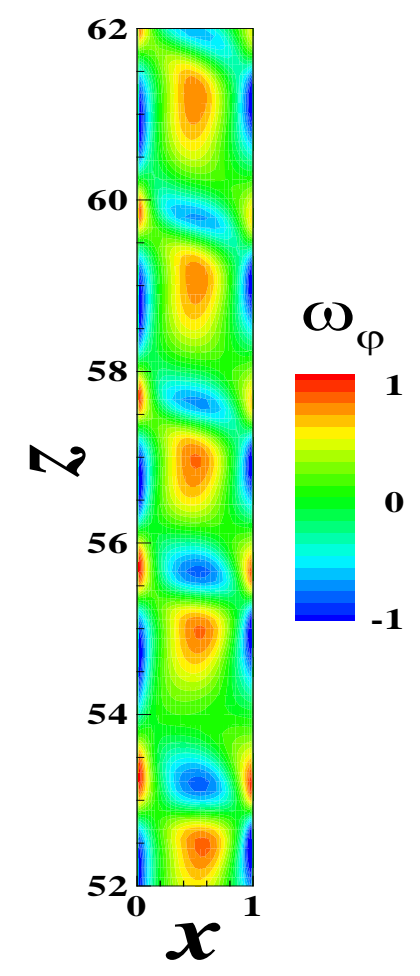

(d)

Figure 4 : Contours of azimuthal vorticity of flow structures in the central part of the flow for $G r$ $=1028$ for different values of $T a:$ (a) $T a=20$, (b) $T a=45$, (c) $T a=75$, (d) $T a=95$. 


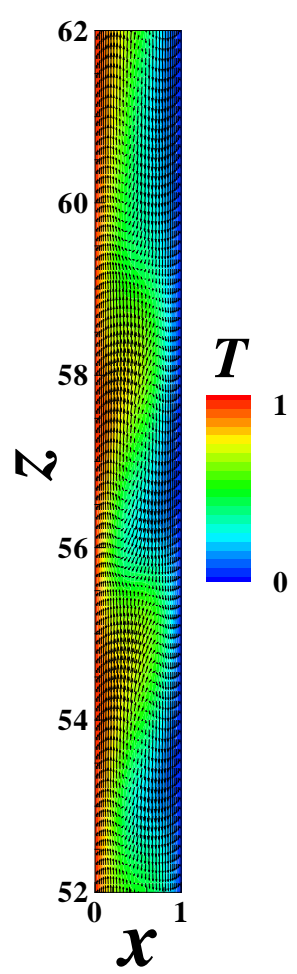

a)

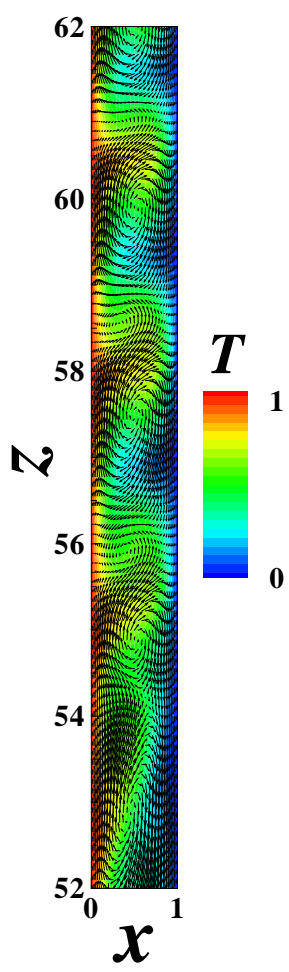

b)

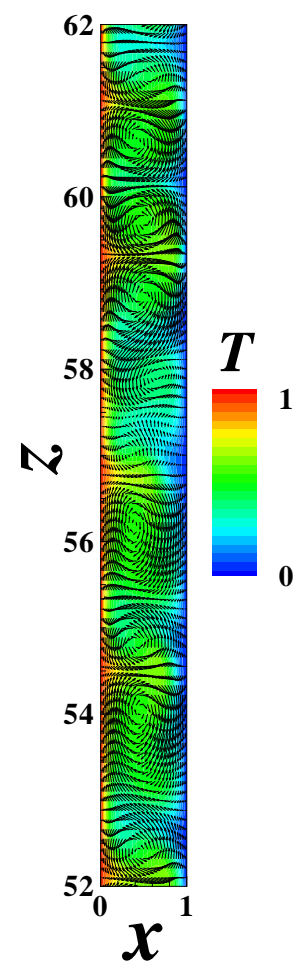

c)

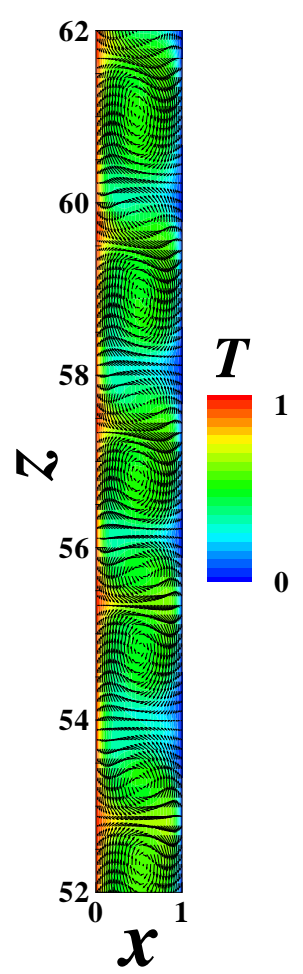

d)

Figure 5: Instantaneous temperature contours and vector plots on $x-z$ plane for $G r=1028$ with different values of $T a:$ (a) $T a=20$, (b) $T a=45$, (c) $T a=75$, (d) $T a=95$.
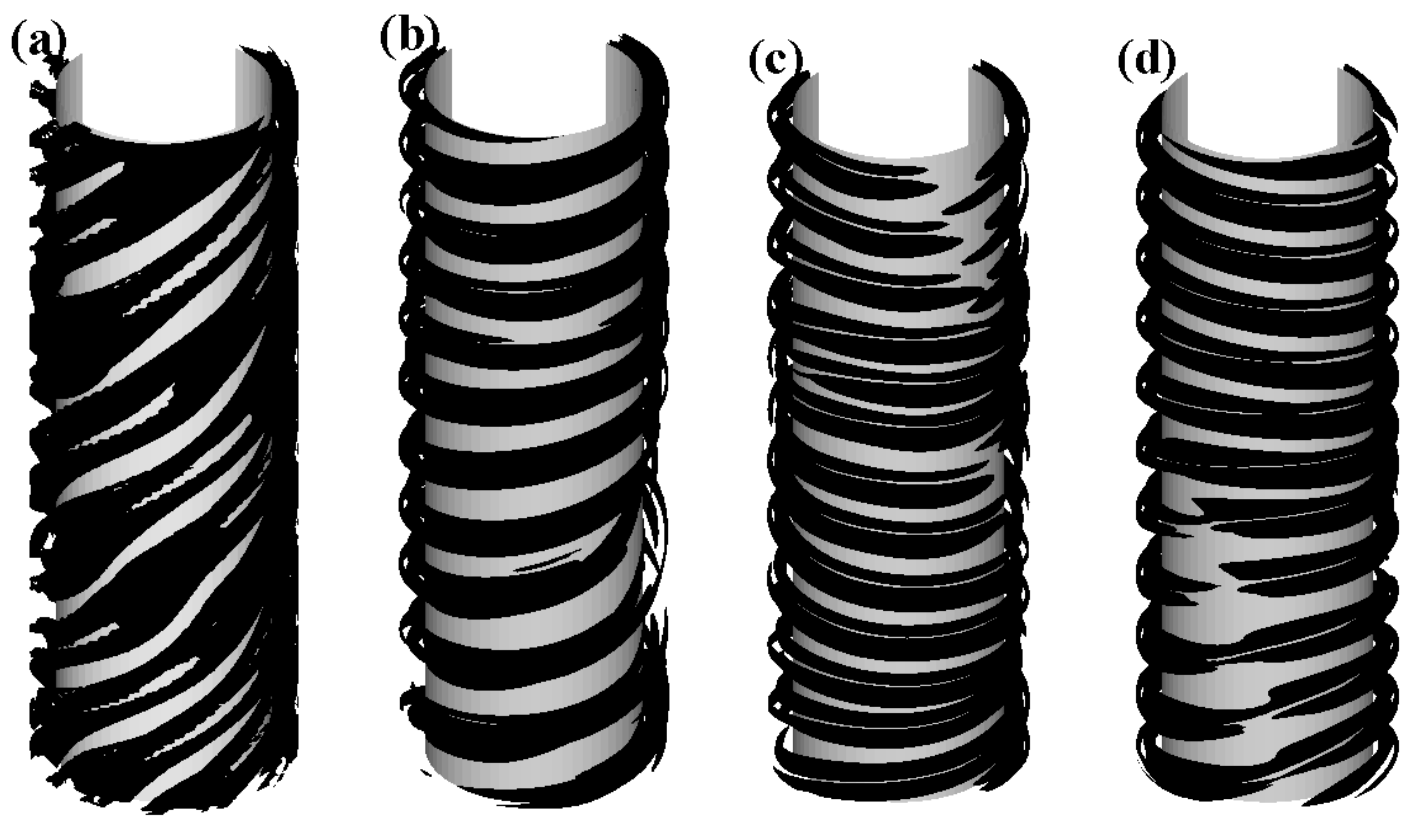

Figure 6: Vortex cores for $\mathrm{Gr}=1028$ and different values of $\mathrm{Ta}: \mathrm{a}) \mathrm{Ta}=20$, b) $\mathrm{Ta}=45$, c) $\mathrm{Ta}$ $=75$, d) $T a=95$. 

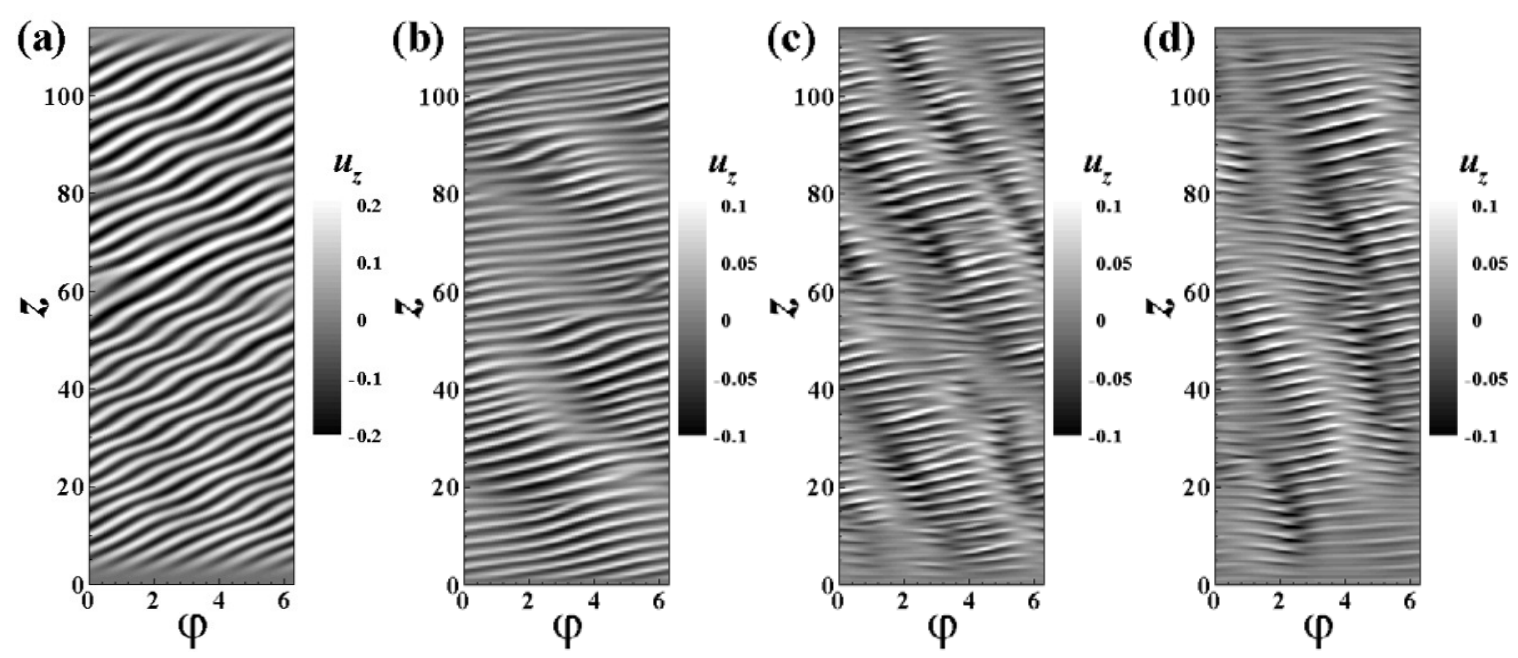

Figure 7: Instantaneous contours of the axial velocity component on the center surface of the annulus $(x=1 / 2)$ for $G r=1028$ : (a) $T a=20$, (b) $T a=45$, (c) $T a=75$, (d) $T a=95$.
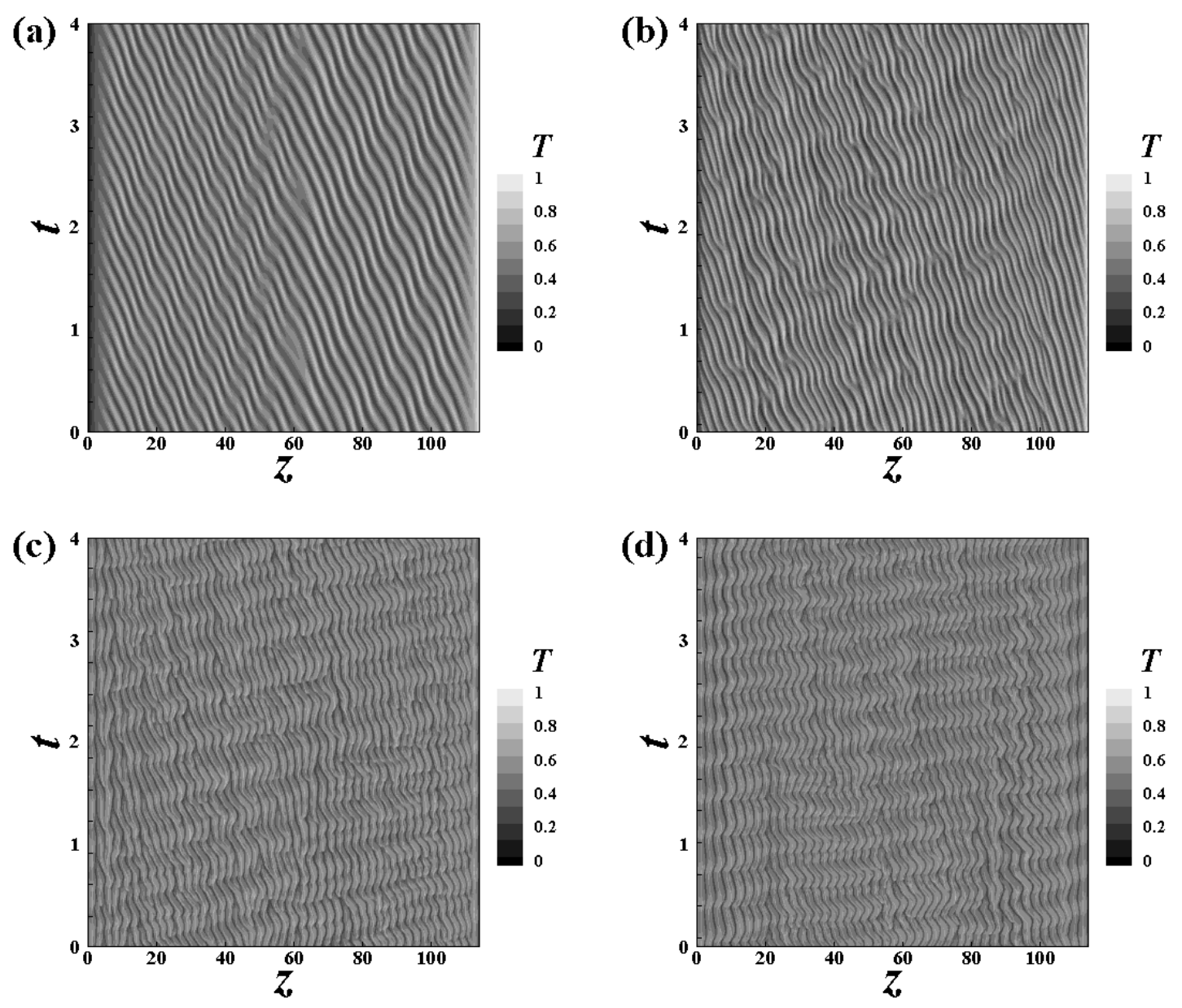

Figure 8: Space-time diagram of the temperature for $G r=1028$ : (a) $T a=20$, (b) $T a=45$, (c) $T a=75$, (d) $T a=95$. 
Following Jeong and Hussain [29], we used the isovalues of the second eigenvalue $\left(-\lambda_{2}\right)$ of the spectrum $\lambda_{i}$ of the symmetric tensor $\overline{\bar{S}}^{2}+\overline{\bar{\Omega}}^{2}$ (with $\lambda_{1}>\lambda_{2}>\lambda_{3}$ ) for identification of vortex cores. Here $\overline{\bar{S}}$ and $\overline{\bar{\Omega}}$ are the rate of strain tensor and the vorticity tensor (i.e. the symmetric and antisymmetric parts of the velocity gradient tensor $\vec{\nabla} \vec{u}$ ) respectively. The cores of the vortex structures are represented in Figure 6 for 4 values of $\mathrm{Ta}$. In Figures 7 and 8 , the instantaneous contours of the axial velocity component and the space-time diagrams of the temperature determined on the center surface of the annulus $(x=1 / 2)$ are shown respectively. The vortices are inclined and travel with a negative phase velocity. While for Ta $=20$, the wave is almost regular (two frequencies), for large values of $T a$, the wave become irregular with the presence of many defects.

\section{Momentum and heat transfer}

We computed the torque exerted on the inner cylinder surface using the relations (6) and (8) of the friction coefficients for fixed value of $G r=1028$ and different values of $T a$. The obtained values $C_{M_{z}}, C_{M_{\varphi}}$ and $C_{M}=\sqrt{C_{M_{z}}^{2}+C_{M_{\varphi}}^{2}}$ are plotted in figure 9.

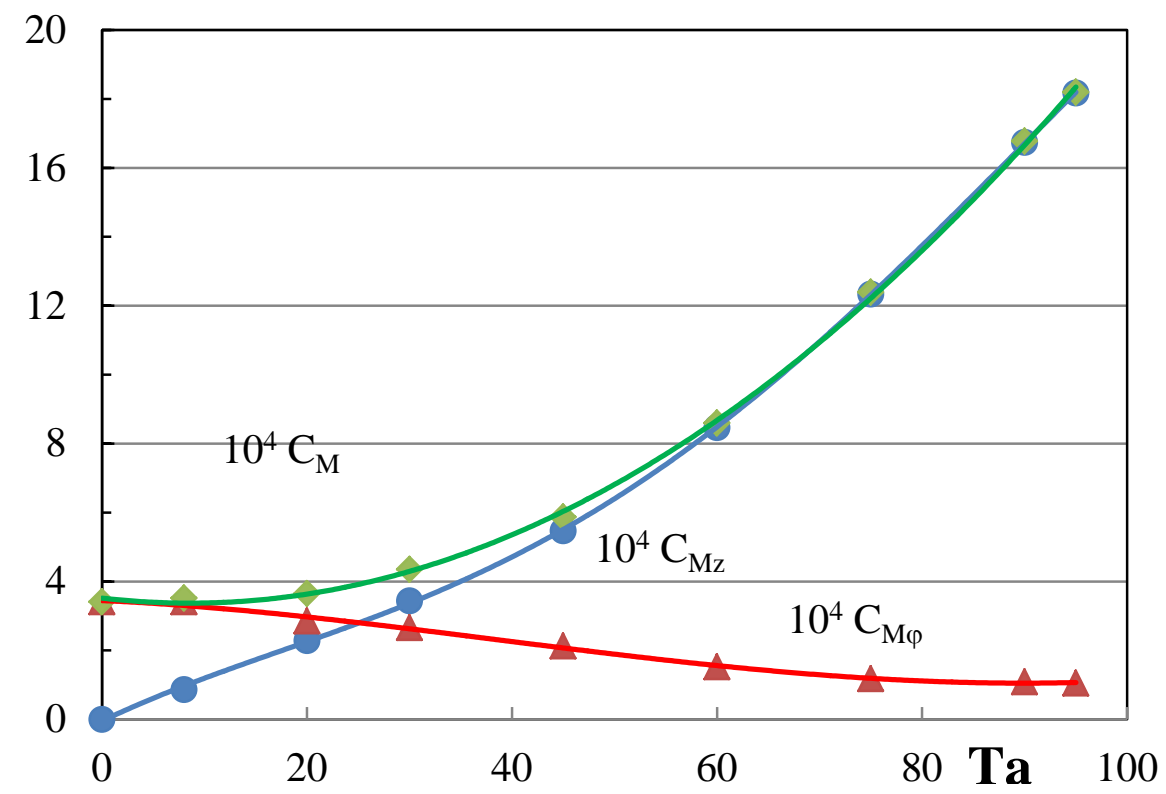

Figure 9: Variation of the friction coefficients on the inner cylinder with the rotation rate (Ta) for $G r=1028$. The coefficients have been multiplied by the factor $(\mathrm{Re} / G r)^{2}$ to ensure the vanishing of $C_{M z}$ for $T a=0$. 
For $T a=0$, the convective cell induced by the temperature gradient exerts a torque on the inner cylinder, the corresponding friction coefficient decreases with Ta.

The torque due to the rotation of the inner cylinder increases with $T a$. For $T a \approx 25$, we found that $C_{M_{z}}=C_{M_{\varphi}}$. For large values of $T a$, the friction coefficient is mainly due to the rotation and the effect of the temperature gradient becomes reduced.

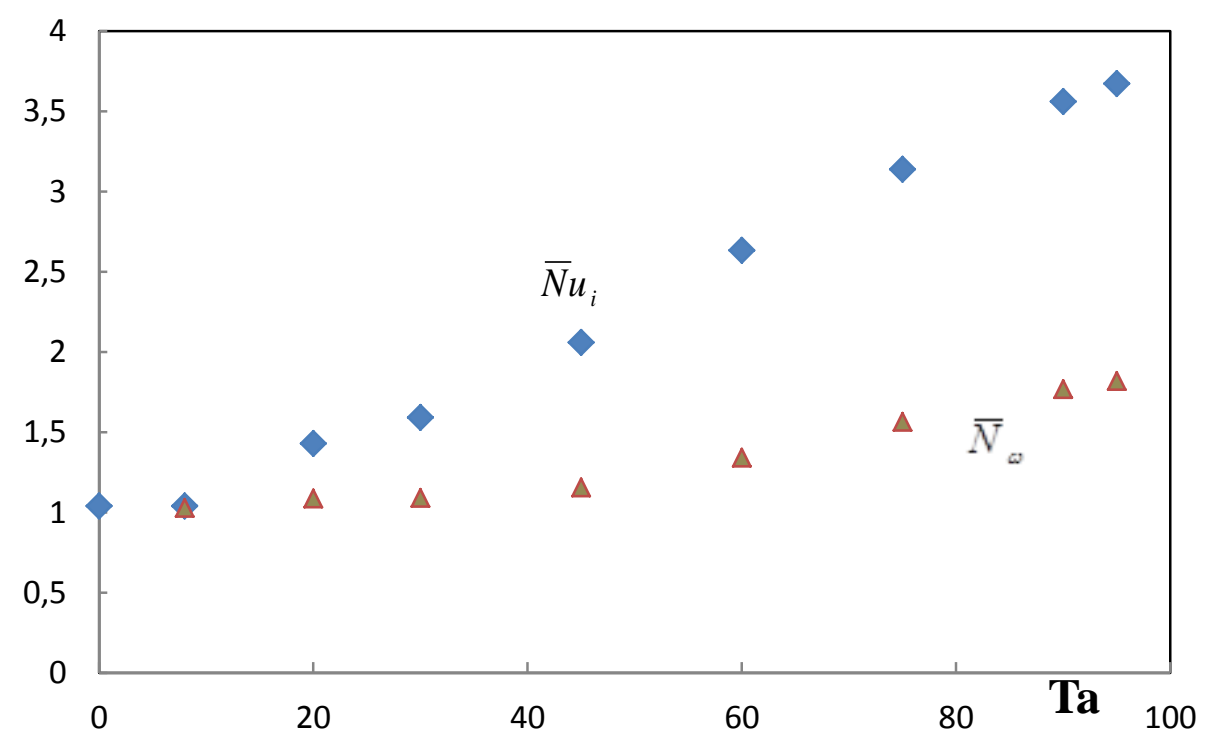

Figure 10: Variation of time-averaged radial transfer coefficients at the inner cylinder with Ta for $G r=1028: \bar{N}_{\omega}$ for the momentum transfer and $\bar{N} u_{i}$ for the heat transfer.

The values of the heat transfer coefficient at the inner cylinder surface have been computed through the Nusselt number using the formula (9). For $\mathrm{Ta} \in\left[0 ; \mathrm{Ta} a_{c}\right]$, the heat transfer is due to the large convective cell and $\bar{N} u_{i} \approx 1$. When Ta is increased above the threshold of the instability, the Nusselt number increases meaning that the secondary vortices induced by the rotation favor the heat transfer (Fig. 10).

Using the formula (10), we have converted the values of the torque into the pseudoNusselt number $N_{\omega}$ plotted in Figure 10. The quantity $N_{\omega}$ is the analogue of the Nusselt number $\mathrm{Nu}$ for the heat transfer and it represents a direct measure of the dissipation rate $\varepsilon$ for isothermal flow : $N_{\omega}=(1+\eta)^{2} \varepsilon / 4$. In the laminar flow $N_{\omega}=1$ for an infinite length annulus and $N_{\omega}>1$ for a finite length annulus; the deviations from 1 of the computed values are due to the endplate effects. There is a net increase of $N_{\omega}$ with $T a$ when $T a>T a_{c}$. ie. the secondary vortex flow favors the radial momentum transfer (Fig. 10). 


\section{EXPERIMENTAL PROCEDURE AND RESULTS}

\section{Experimental setup and procedure}

The experimental setup consists of two coaxial cylinders of height $H=55.9 \mathrm{~cm}$ and a gap $d=0.5 \mathrm{~cm}$. The aspect ratio of the system is $\Gamma=111.8$ and the radius ratio $\eta=0.8$. The experimental setup has been described in [21]. Inside the inner cylindrical tube, water maintained at controlled temperature $T_{1}$ circulates and the outer cylinder is immersed in a large thermal bath maintained at controlled temperature $T_{2}$. The working fluid is a deionized water with a kinematic viscosity $v=10^{-2} \mathrm{~cm}^{2} / \mathrm{s}$ at $T=293 \mathrm{~K}$. Few experiments were run with a mixture of $50 \%$ water-glycerol in order to obtain very viscous solutions and then to reach small values of $G r$ for the working temperature range of the thermostats.

The length of the annulus has been chosen in order to ensure that the base state is a laminar conduction regime for weak values of the temperature difference in the range of the Prandtl numbers admissible in the experiment [16, 30]. In our experiments, the temperature difference intervals were chosen such that the average temperature varies between 293 and $313 \mathrm{~K}$, so that the values of $\operatorname{Pr} \in[4 ; 7]$. According to de Vahl Davis and Thomas [12], the laminar conduction regime exists if $R a=G r \operatorname{Pr}<400 \Gamma$. For our flow configuration, this leads to $R a<44000$ or $G r<11000$.

The flow was visualized by adding $2 \%$ by volume of Kalliroscope AQ1000 which is a suspension of $1-2 \%$ of reflective fin flakes $(30 \mu \mathrm{m} \times 6 \mu \mathrm{m} \times 0.07 \mu \mathrm{m})$. These flakes have a large reflective optical index $n_{\text {opt }}=1.85$ and a density $\rho=1.62 \mathrm{~g} / \mathrm{cm}^{3}$. The sedimentation of these particles remains negligible in vertical configuration if the flow height is not too large and the experiment lasts less than $10 \mathrm{~h}$ [31]. In fact their sedimentation velocity in water at $22^{\circ} \mathrm{C}$ is $\mathrm{v}_{\mathrm{s}}=2.8 \times 10^{-5} \mathrm{~cm} / \mathrm{s}$ [32]. The Kalliroscope particles in small concentrations $(<5 \%$ by volume) do not alter the flow viscosity. We have tested the stability of the Kalliroscope suspension with the temperature variation within the working temperature interval and we found that no aggregates were formed as long as the temperature difference imposed on the solution was less than $35^{\circ} \mathrm{K}$. For water-glycerol mixtures, the precision on $G r$ is $\Delta G r= \pm 10$ (for $\mathrm{Gr}<150)$; while for deionized water, $\Delta G r= \pm 50(G r>200)$. 

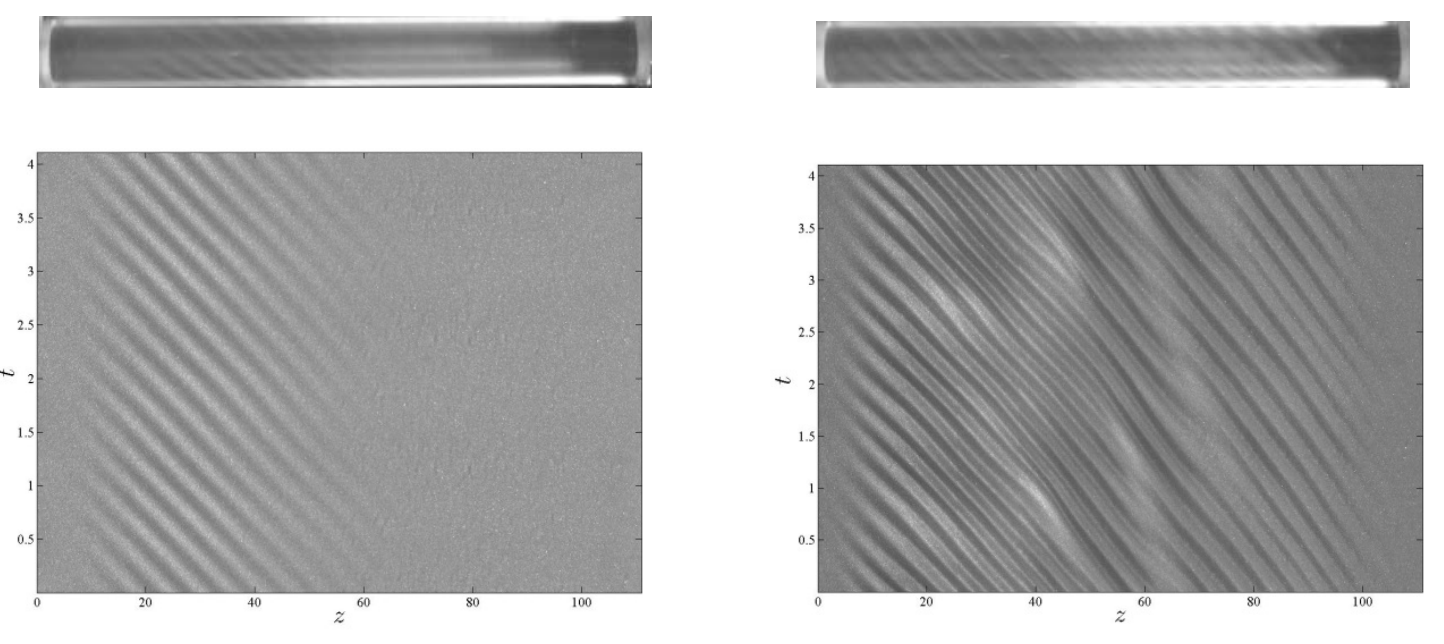

a)

b)
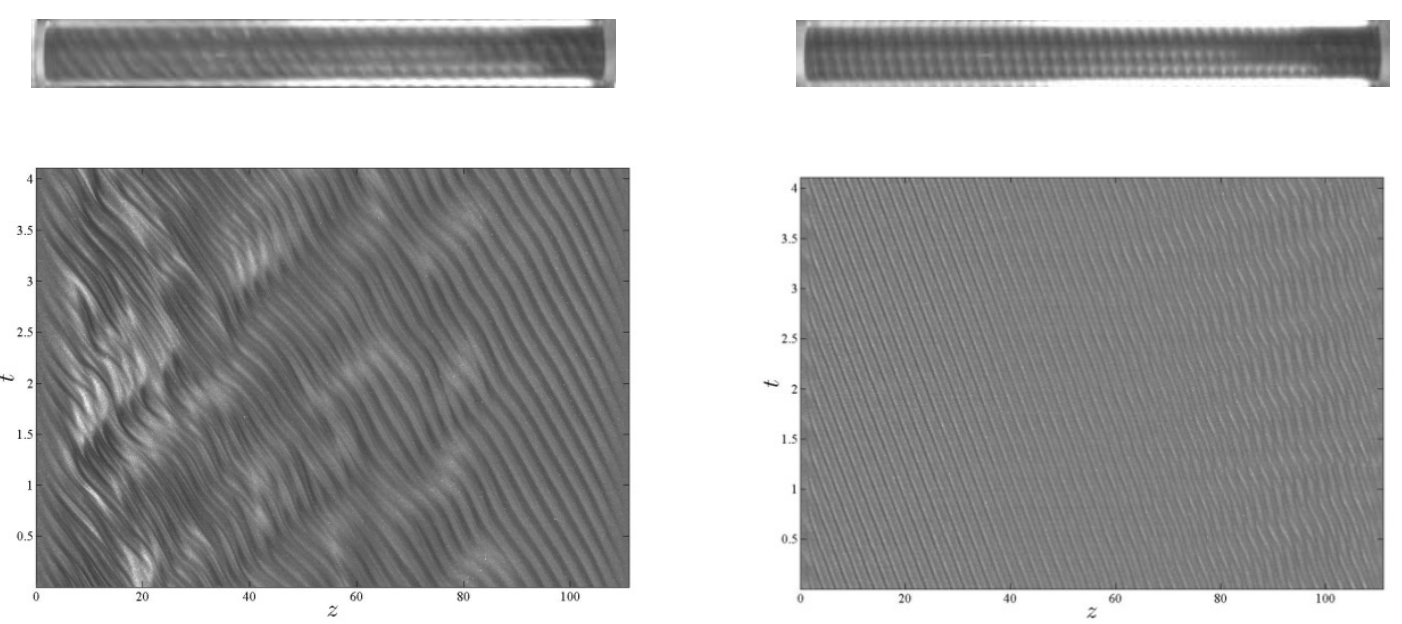

c)

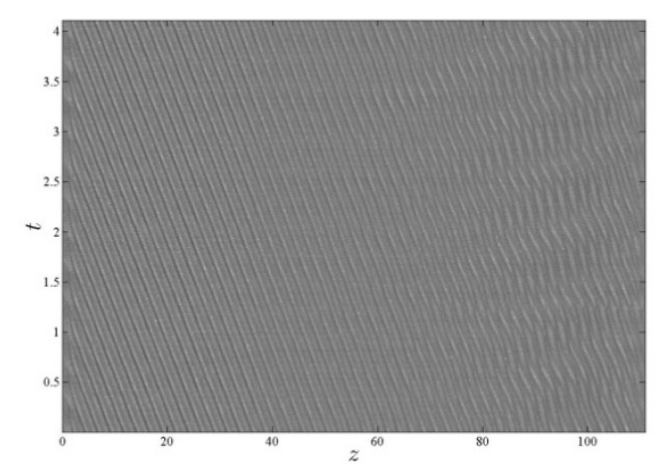

d)

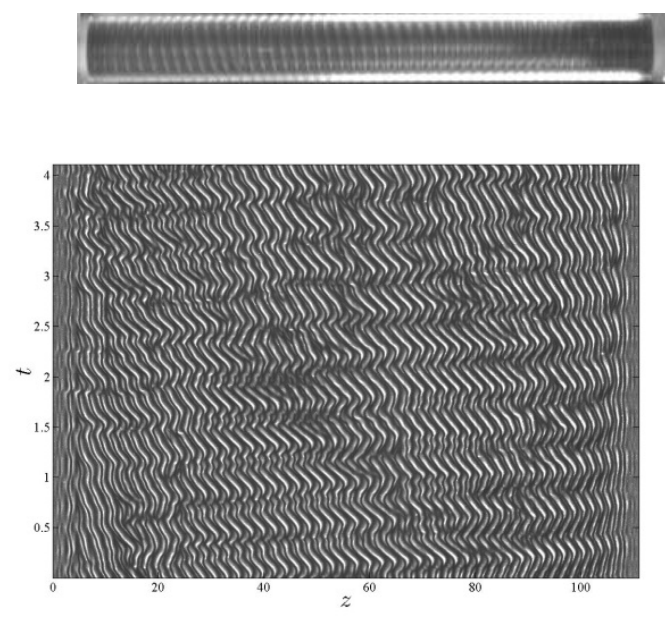

e)

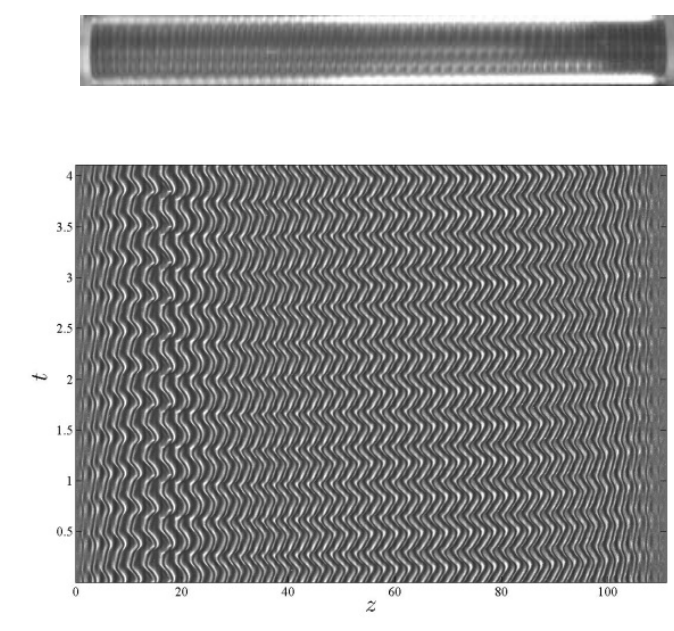

f)

Figure 11: Different states observed for $\mathrm{Gr}=1028: \mathrm{a}) \mathrm{Ta}=10.6$, b) $\mathrm{Ta}=12.3$, c) $T a=20$, d) $T a=45$, e) $T a=75$, f ) $T a=95$. 
In order to detect different transitions in the flow patterns, we fixed the temperature difference (i.e. $G r$ ) between the cylinders and then we increased the rotation of the inner cylinder in a quasi-static way from the base flow state to higher states. For each regime, the waiting time between changes of $\Omega$ was at least 20 minutes which was found to be large enough to ensure the established flow for the states under investigation. We waited 30 minutes before data records for each flow state. The flow was visualized in the meridional plane (r-z plane). We used a He-Ne laser beam through a cylindrical lens which generated a plane vertical beam parallel to the cylinder axis over the whole working length. At regular time intervals, a linear CCD camera was used to record the intensity distribution of light reflected by Kalliroscope particles. Typical flow patterns observed in the experiment are shown in Figure 11. The intensity distributions along a vertical or horizontal line in the meridional plane were superimposed at regular time intervals to construct space-time diagrams $I(\mathrm{z}, t)$ (Fig.11). The spectral analysis of the signals $I(\mathrm{z}, \mathrm{t})$ yields the axial wavenumber $q$ and the frequencies $f$ of the flow regimes. The azimuthal wavenumber $n$ was determined from the inclination angle of vortices : $\phi=\tan ^{-1}\left(k_{\phi} / q\right) ; k_{\phi} \equiv 2(1-\eta) n /(1+\eta)$.

\section{$\underline{\text { A. Experimental Results }}$}

In the experiment, the temperature difference imposed on the annulus $(G r)$ is fixed and the rotation is progressively increased by step of $\Delta \mathrm{f}=1 \mathrm{mHz}$ corresponding to $\Delta T a=0.4$ from the laminar state to different higher states (Fig. 12).

\section{A1. Primary instability modes}

The states observed when the flow bifurcates from laminar base flow can be gathered into four groups. The first group consists of stationary axisymmetric vortices that are observed for small values of $G r$ (i.e. $|G r|<30$ ). These are the Taylor vortices, their threshold and their wavenumber are almost constant with respect to $G r$. The second group appears for

]-800;-30[U]30;1100[ : the critical state appears first as partial spiral vortex flow localized in the neighborhood of the bottom endplate (Fig.11-a). Their spatial extent increases when $T a$ is slightly increased until the spiral pattern fills the entire system (Fig.11-b). These states were described in detail in [21]. The threshold and the wavenumber of these states decrease with $G r$ while their frequency increases. The direction of their propagation is determined by the helicity which is proportional to the product $G r T a$. The third group appears for ]-9000;-800[U]1100;9900[ where the critical state occurs in the form of a modulated wave. Within the precision of the experiment $\delta T a=0.4$, it was impossible to 
detect any non-modulated state (single wave) before the modulated pattern appears in the flow.

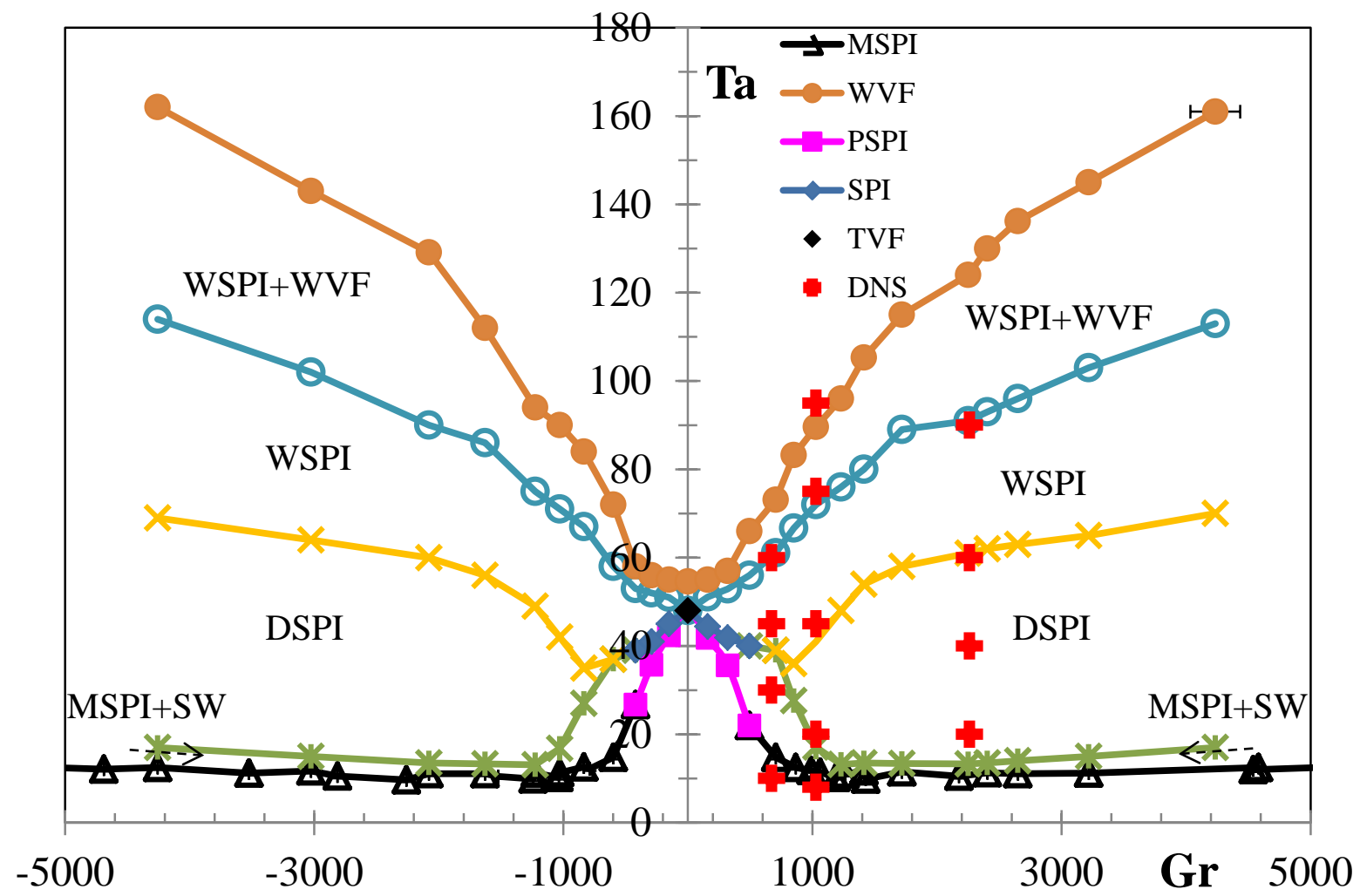

Figure 12: State diagram of the flow patterns observed in experiment in the space $(\mathrm{Gr}, \mathrm{Ta})$ : SPI : Spiral pattern, PSPI (Partial Spiral Pattern), MSPI (Modulated Spiral Pattern), DSPI (Disordered Spiral Pattern), WSPI (Wavy Spiral Pattern), TVF (Taylor Vortex Flow), WVF ( Wavy Vortex Flow), SW (Solitary wave). The crosses represent computed states in the DNS.

The threshold and the axial wavenumber are almost independent of $G r$. The fourth group occurs for $-8800<G r$ and $G r>9900$ where the critical states appear for $T a=0$, the critical modes correspond to natural convection in a cylindrical annulus and originate from the destabilization of the large convective cell yielding secondary vortices as reported in [30].

\section{$\underline{\text { A2. Higher instability modes }}$}

In this section, we describe the flow states observed for $G r<4500$; the higher states for $G r>4500$, have not been explored yet. The transition to higher instability modes is very sensitive to the values of Gr. For $G r<30$, the secondary instability leads the Taylor vortex flow (TVF) to a wavy vortex flow (WVF). For $30<G r<1100$, the spiral pattern (SPI) bifurcates to a wavy spiral pattern (WSPI) and then to a state which is a mixture of wavy spiral and wavy vortex flow, before becoming completely a WVF. 


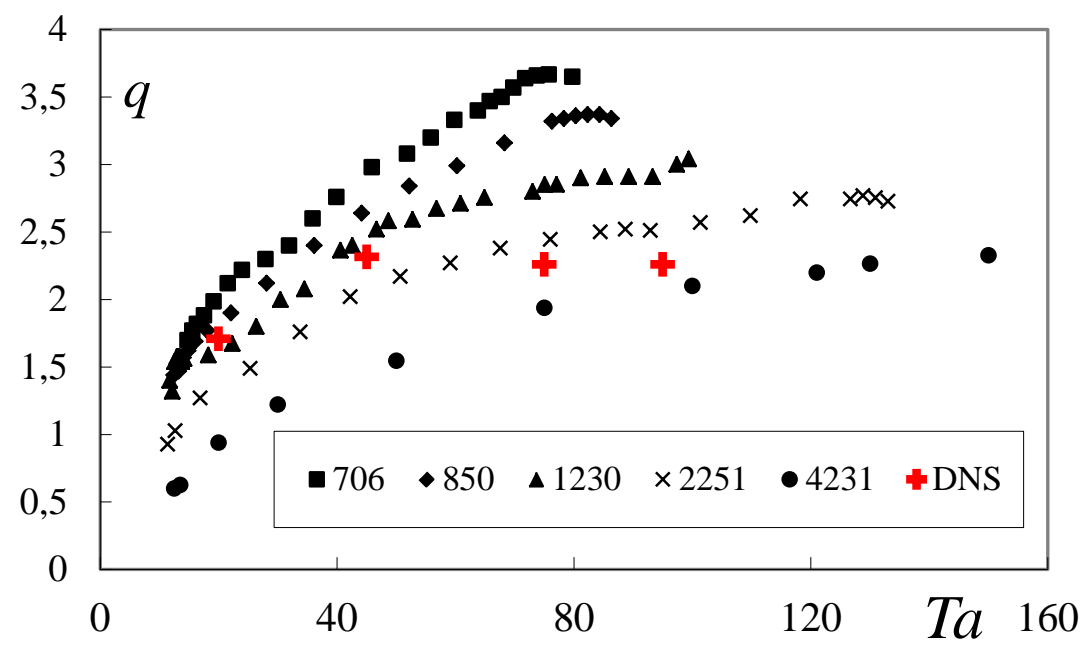

a)

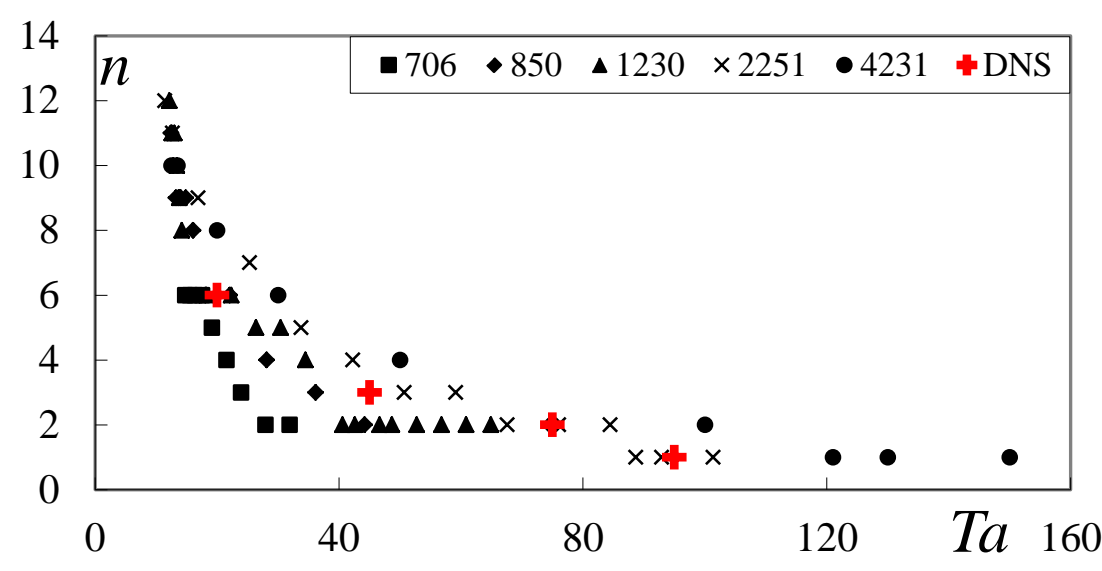

b)

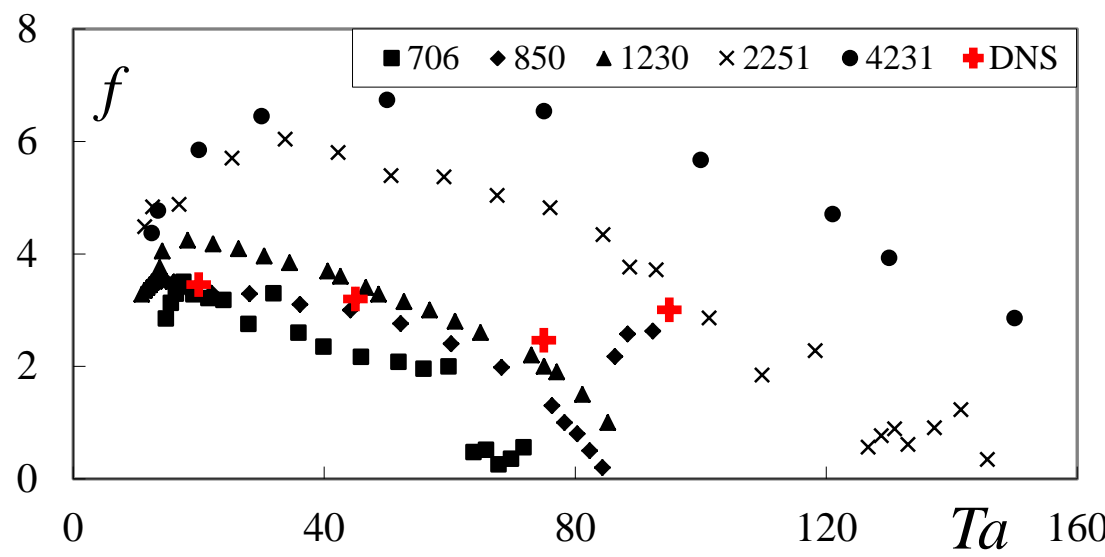

c)

Figure 13: Variation of spatio-temporal properties of the patterns with $T a$ for different values of $G r$ : a) axial wavenumber ( $q$ ), b) azimuthal wavenumber ( $n$ ) and c) frequency. DNS points correspond (crests) to $G r=1028$ and $T a \in\{20,45,75,95\}$ 
For $1100<G r<2500$, the modulated spiral pattern (MSPI) bifurcates to a state of disordered spiral patterns (DSPI) which becomes wavy spiral pattern (WSPI) and then to a mixture of wavy spiral and wavy vortex flow as $T a$ increases. For $G r>2500$, within an increase of $\delta T a=0.4$, a solitary wave (SW) appears on the background of the modulated spiral pattern (MSPI). This solitary wave is a robust structure and persists even when $\mathrm{Ta}$ is increased. A typical transition from the base flow state to the wavy vortex flow is illustrated in Figure 12 for $G r=1028$.

We found that for each value of $G r$, the transition to wavy vortex flow occurs when the value of $R i$ becomes close to 0.03 , ie. when the effects of rotation become sufficiently dominant over the thermal effects. The variations of the axial, azimuthal wavenumbers and the frequency of the different flow patterns with $G r$ are given in Figure 13. For a fixed value of $G r$, the axial (azimuthal) wavenumber increases (decreases) with Ta towards the value of the wavy vortex flow. The frequency increases, reaches a maximum and then decreases to the value of the wavy vortex flow.

\section{DISCUSSION}

The experimental diagram of configuration states (Fig. 12) is almost symmetric with respect to the inversion of the sign of $G r$ i.e. almost independent of the heating or cooling of the inner or the outer cylinder. This symmetry can be explained by the independence of the first instability modes on the sign of $\mathrm{Gr}$ as predicted by the linear stability analysis of Yoshikawa et al. [23] for small values of $G a$. In fact, we have used an experimental setup with $G a^{-1 / 2} \in[2 ; 4] \times 10^{-4}$ which is very small and the centrifugal buoyancy has no significant effect. The flow system possesses the symmetries $S^{c / r}(\Omega)$ and $S^{c / r}(\Delta T)$ described by Ali and Weidman [19]. However, one should keep in mind that in an experiment, there is a small dissymmetry due to the difference of thermal properties in the experimental setup: the inner cylinder is made of aluminium while the outer cylinder is made of glass, so both materials have different heat conduction coefficients. For simplicity, we will discuss only the results for positive values of $G r$. 


\section{Variation of the threshold with Gr}

The isothermal value of $T a_{c}$ was used to validate the numerical code: $T a_{c}^{L S A}=47.34 ; T a_{c}^{\exp }=T a_{c}^{D N S}=48$. Figure 14 shows the variation of the critical values $T a_{c}$ with $G r$. The values obtained by linear stability analysis (LSA) and DNS are in good agreement with the experimental values for $G r<3000$. When the temperature difference is imposed on the annulus, the main result is that, except for very small values of $G r$ where the threshold is almost constant and the critical mode is stationary, the threshold of instability decreases with $G r$ i.e. the radial temperature gradient destabilizes the flow, and the critical modes are time dependent (i.e. the transition is a Hopf bifurcation). This experimental result has been obtained in previous studies $[16,17,21]$ which were limited to small values of $G r$.

The main mechanisms of destabilization are the centrifugal force, the Archimedean buoyancy and the shear force from the baroclinic velocity induced by the temperature radial gradient. Energetic analysis performed in [23] for a flow system with a radius ratio $\eta=0.8$, has shown the existence of 4 ranges for $\mathrm{Gr}$ corresponding to different contributions: for very weak values of $G r(G r<22.5)$, there is no buoyancy power input; centrifugal force is the only dominant mechanism leading to stationary axisymmetric vortices. As $G r$ increases, the power produced by Archimedean buoyancy increases and that of the centrifugal force decreases, but both these power contributions favor instability leading to the decrease of the threshold and to the occurrence of oscillatory non axisymmetric modes that manifest themselves as spiral or helicoidal vortices. For $G r>1500$, the power from centrifugal force almost vanishes while that from the Archimedean buoyancy reaches its maximum value. In this case, only Archimedean buoyancy produces flow destabilization. The critical modes are time-dependent non axisymmetric modes that appear in the form of the waves. The fourth zone is the natural convection that occurs for $T a=0$ and $G r=7960$. This instability mode is called hydrodynamic mode (HM) because it is driven by the shear of the axial velocity profile which possesses an inflexion with a maximum of azimuthal vorticity [15]. According to the Rayleigh-Fjörtföt criterion, the flow is unstable and leads to transverse vortices which are oscillatory because of the curvature.

All the patterns are either stationary or periodic in time, so that averaging over a period of oscillations, the l.h.s. of the equation (2) vanishes. As the centrifugal buoyancy is negligible in our system, there is a balance between the term containing the torque due to the rotation, the term due to the buoyancy production of the power and the dissipation term. For small values of $G r$, the buoyancy production is very weak and only the torque produces 
energy to amplify flow perturbations. For large values of $G r$, the buoyancy power is large enough so that a small amount of torque triggers the thermally-driven perturbations.

We should remember that in our experiment, for $G r<1100$, the transition occurs via a convective instability, i.e. the pattern is formed near the bottom endplate and then increases in size until it fills the whole flow system as Ta is increased [21]. The present DNS could not retrieve this partial spiral pattern which is related to the sensitivity of the experimental system.

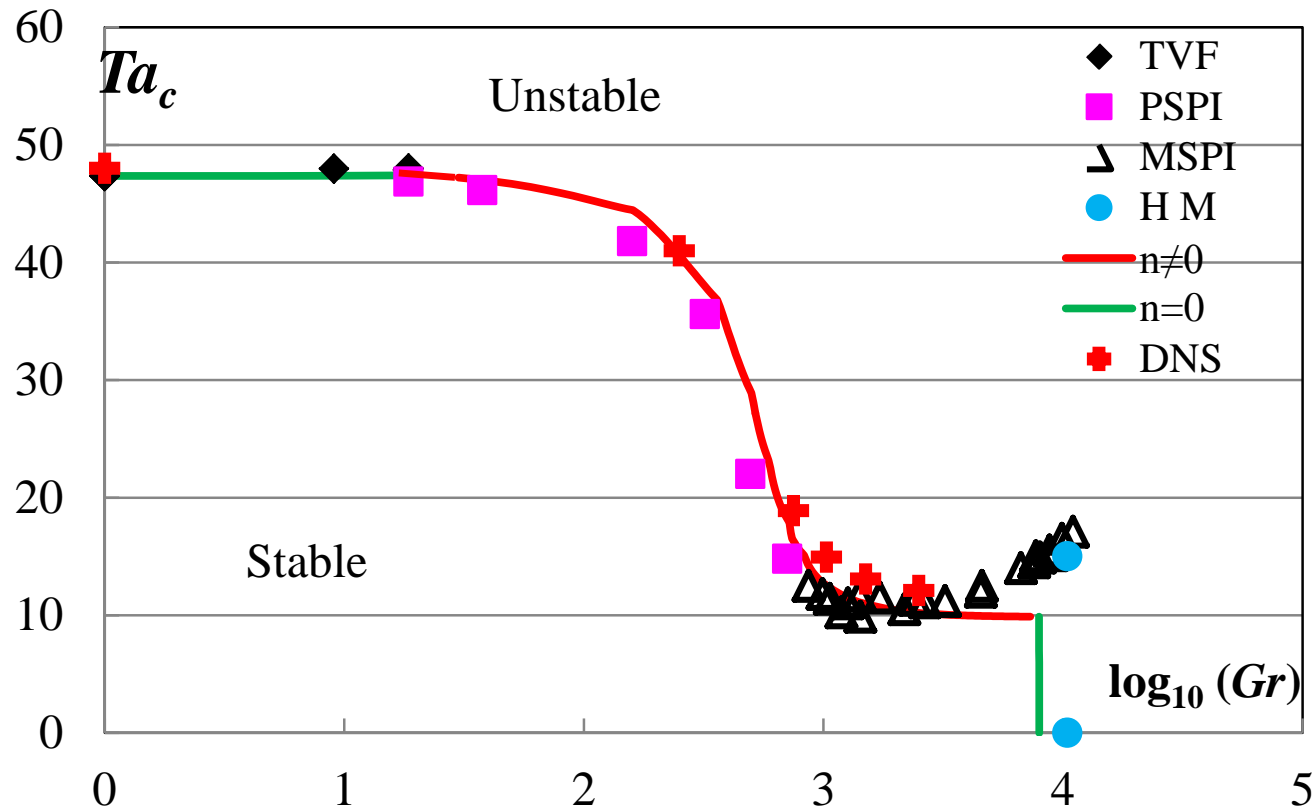

Figure 14: Variation of the threshold $\mathrm{Ta}_{c}$ with $\mathrm{Gr}$ : comparison between results from LSA (continuous line, [23]), DNS (crosses) and experiment. Labels as in Figure 12.

\section{Non-Boussinesq effects}

For $G r>3000$, there is a noticeable deviation between experimental and computed values (Fig.14). This discrepancy can be explained by the non-validity of the Boussinesq approximation which assumed that the temperature difference is too small to consider the fluid density as a linear function of the temperature only in the terms responsible of instability and a constant in all other terms. In our experiment, this assumption becomes non valid for $G r>3000$. In fact, the measurement of the axial variation of the temperature showed that above this value of $G r$, there is a significant vertical temperature gradient [33]. According to Bergholz [34], the vertical temperature gradient can be neglected if the temperature stratification parameter $\gamma=(\operatorname{Ra} \tau / 4)^{1 / 4}<2$ where $\tau=(d T / d z)(d / \delta T)$. For our experiment, this condition is satisfied for $-2000<G r<3000$. For values of $G r$ outside of this interval, the Boussinesq approximation is not valid and this is the reason for discrepancy between experimental and linear stability analysis data. This situation can be exemplified for natural 
convection [35]: while the linear stability predicts $G r_{c}=7960$ for the occurrence of a hydrodynamic mode, the experimental value of $G r$ is $G r_{c} \approx 9800$. The difference is considerable.

\section{$\underline{\text { Vortex size and frequency }}$}

The presence of a weak temperature gradient in the flow confined in a differentially rotating annulus induces an increase of the axial wavelength, the azimuthal and the frequency until the occurrence of the modulated wave, i.e. for $\mathrm{Gr} \approx 1100$.

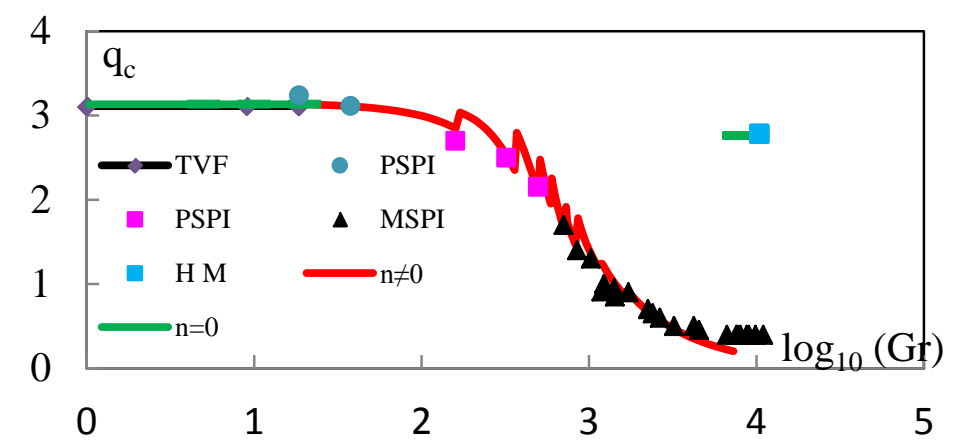

a)

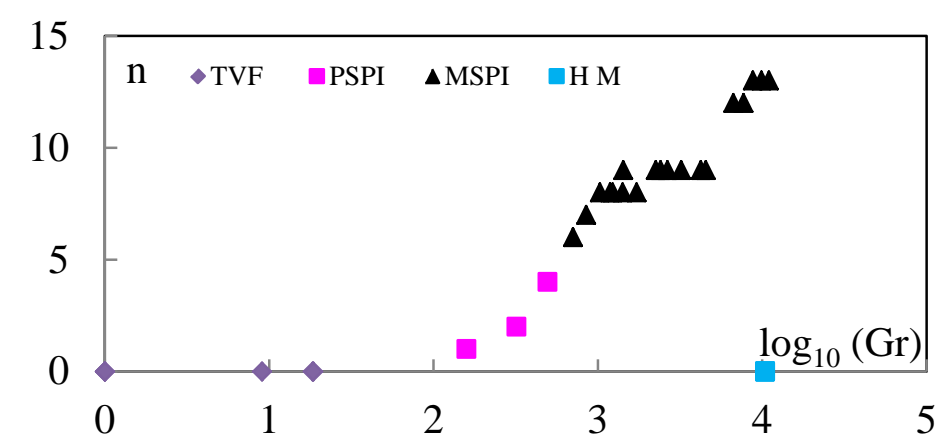

b)

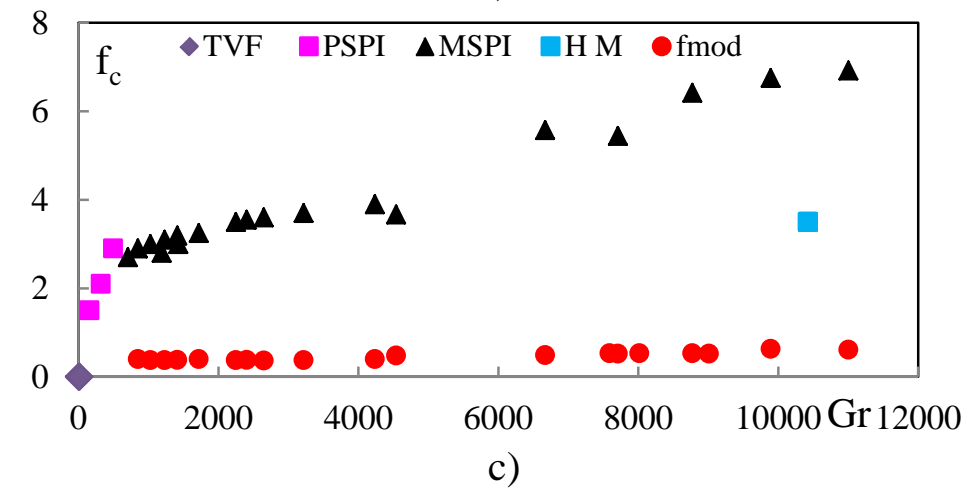

Figure 15 : Variation of the critical parameters with $G r:$ a) axial wavenumber, b) azimuthal wavenumber, c) frequency. Labels as in Figure 12. Continuous line in a) corresponds to results from LSA. 
For large values of $G r$, there is no increase in wavelength, but the azimuthal wavenumber and main frequency still increase. The pattern is modulated and the modulation frequency is almost independent on $G r$.

\section{$\underline{\text { Structure of the flows }}$}

The meridional cross-sections of the flow patterns obtained in experiments and in DNS show the difference between the critical regimes observed for low values and for large values of $G r$. In fact, for low values the SPI are corotating vortices while for large values the critical states are modulated waves. For a fixed value of $G r$, i.e. for a fixed strength of the base convective cell, the increase of the rotation rate leads to the transition from corotating vortices or modulated waves to counter-rotating vortices, this transition depends on Ta. This transition is due to the competition between Archimedean buoyancy and centrifugal force due to the rotation. This competition is described by the Richardson number $R i$ which measures the relative importance of the buoyancy and rotation effects. The data corresponding to these transitions in three systems are given in Table 1 where $R i_{c}$ corresponds to the threshold, $\mathrm{Ri}^{+}$to the transition from corotating vortices or modulated waves to counter-rotating vortices and $R i$ * to the transition to wavy vortex state. The transition to wavy vortex flow was observed in our experiment for $R i^{*} \approx 0.03$.

Table 1 : Values of $R i$ at which there are transitions for different studies.

\begin{tabular}{|l|c|c|c|c|}
\hline \multicolumn{1}{|c|}{ Study } & $G r$ & $R i_{C}$ & $R i^{+}$ & $R i^{*}$ \\
\hline Present experimental study & 706 & 0.784 & 0.058 & 0.036 \\
\cline { 2 - 5 } $\operatorname{Pr} \approx 5.4, \eta=0.8$ & 1028 & 1.815 & 0.153 & 0.032 \\
\cline { 2 - 5 } & 2405 & 4.969 & 0.587 & 0.031 \\
\hline $\begin{array}{l}\text { Ball } \text { et al. [18], Pr =0.7 } \\
\eta=0.437,0.565,0.656\end{array}$ & 1000 & 0.237 & 0.156 & 0.10 \\
\cline { 2 - 5 } $\begin{array}{l}\text { Ali \& Weidman [36] } \\
\operatorname{Pr}=21, \eta=0.62\end{array}$ & 22056 & 0.31 & 0.25 & 0.12 \\
\hline
\end{tabular}

The inner structure of the WVF in the presence of temperature gradient is more complicated. In fact, the temperature measurements have shown that thermal boundary layers exist near the cylindrical surfaces with strong gradients of the temperature [37]. In the experiment of Ball et al. [18] with $\eta=0.5$ and $\operatorname{Pr}=0.7$, the transition was found for $R i^{*}=0.12$ 
but the final vortex state was the Taylor Vortex Flow. This result was confirmed in the DNS of Kuo \& Ball [20]. We suspect that the last study did not observe wavy vortex flow because of the short aspect ratio used in their experiment and in numerical simulations. The data in Table 1 indicate that the value of $\mathrm{Ri}^{*}$ for the transition to wavy vortex flow depends on $\mathrm{Pr}$ but it is insensitive to the radius ratio $\eta$.

\section{$\underline{\text { Momentum and heat transfer }}$}

The torque on the inner cylinder has been expressed through the pseudo-Nusselt number $N_{\omega}$ which is almost constant $\left(N_{\omega} \approx 1\right)$ for $T a<T a_{\mathrm{c}}$ and increases with $T a$ in the supercritical regime [38]. Present DNS show that $N_{\omega}$ is not sensitive to $G r$ (Fig. 9). The temperature gradient has a very weak effect on momentum transfer which is ensured by the rotation.

Besides $N_{\omega}$, we have determined the momentum transfer through the friction coefficient $C_{M}$ for which there are few correlation formula for the Couette-Taylor flow for $T a>T a_{c}$. One of them is the Bilgen-Boulos correlation [2] which when applied to our flow system with $\eta=$ 0.8, yields $C_{M}=2^{-0.2} \mathrm{Ta}^{-0.6}$ leading to larger values than our computed values.

The Nusselt number $\mathrm{Nu}$ increases with $\mathrm{Ta}$ by a factor from $\mathrm{Ta}=0$ to $\mathrm{Ta}=100$. This increase is a signature of the main action of the secondary vortices induced by the rotation in the enhancement of the heat transfer. In fact, these secondary vortices transfer heat directly in the radial direction from the hot wall to the cold one along the shorter path than that of the larger convective cell. We have compared the computed values with the different correlations $N u=f(\eta ; T a, G r, \operatorname{Pr})$ that are available in the literature [4]. The best correlation for our data for $G r=1028$ is given by the Aoki et al. [39] with $N u=0.22 \mathrm{Ta}^{0.5} \operatorname{Pr}^{0.3}$. The correlations suggested recently in [40] are limited to few values of $R a=G r \operatorname{Pr}$ and conclude the insensitivity of correlation with $R a$. This seems to be in contradiction with other available results [24] which reported different values of $\mathrm{Nu}$ for $\mathrm{Ta}=40$ and varying $\mathrm{Gr}$. Therefore, there is a need for a thorough experimental and numerical work to update the available correlations.

\section{CONCLUSION}

We have performed a thorough investigation of stability of the flow between differentially rotating annulus with a radial temperature gradient for a sufficiently large interval of data compared to the previous studies. The base flow is a combination of the circular Couette flow and a vertical baroclinic flow ascending near the hot surface and 
descending near the cold one. The first instability modes are stationary axisymmetric vortices for a very weak temperature gradient, traveling helicoidal vortices for intermediate values of $G r$ and modulated waves for large values of $G r$. While in experiments, large values of $G r$ up to 10000 were explored at least for the first instability, in DNS we were limited to those values of $\mathrm{Gr}$ for which the Boussinesq approximation was still valid (i.e. $G r<3000$ ). A diagram of different flow regimes observed for $T a<170$ and $|G r|<5000$ is established. The competition between the thermal and rotation effects depend on Richardson number Ri. We found that for our experimental system, the effects of the temperature gradients are confined near the cylindrical surfaces when $R i$ decreases below $R i^{*}=0.03$. For $|G r|<3000$, we have found a good agreement between experimental results, those from linear stability analysis and from numerical simulations. The heat transfer and the torque increases as the rotation rate increases due to the secondary vortices. However, the rotation has a stronger effect on the heat transfer than on the torque transfer in the radial direction. For applications, there is a need for the exploration of a larger range of ( $\mathrm{Ta}, \mathrm{Gr})$ in order to obtain accurate correlations.

\section{Acknowledgments}

The authors are thankful to D. Hallidy for the help in the reading of the manuscript. This work was initiated by bilateral exchange programs STAR and CNRS-KOSEF between France and Korea. K.S Y. was also supported by the National Research Foundation of Korea (NRF) grant funded by the Korean government (MSIP) (No. 2015R1A2A2A01002981). R.G. acknowledges a scholarship from the Regional Council of Haute-Normandie. LOMC researchers acknowledge the financial support from European Regional Development Fund (FEDER), the CPER-Haute Normandie under the programme THETE and from the French National Research Agency (ANR) through the program Investissements d'Avenir (ANR-10 LABX-09-01), LABEX EMC ${ }^{3}$ (project TUVECO). I.M. thanks Patrick Weidman for providing him with unpublished data from his experiment. 


\section{REFERENCES}

[1] F. Kreith, Convection heat transfer in rotating systems, in Advances in Heat Transfer 5, 129-251 (1968). Academic Press, New-York.

[2] P.R.N. Childs, Rotating Flow, Butterworth-Heinemann (2010).

[3] F. Tachibana, S. Fukui and H. Mitsumura, Heat transfer in an annulus with an inner rotating cylinder, Bulletin of the JSME (Japan Society of Mechanical Engineers) 3 (9), 119123 (1960).

[4] Y.N. Lee and W.J. Minkowycz, Heat transfer characteristics of annulus of two coaxial cylinders with one cylinder rotating, Int.J. Heat Mass. Transfer 32, 711-722 (1989).

[5] M. Fénot, Y. Bertin, E. Dorignac and G. Lalizel, A review of heat transfer between concentric rotating cylinders with or without axial flow, Int. J. Thermal Sci. 9, 1138-1155 (2011).

[6] R. Kedia, M.L. Hunt and T. Colonius, Numerical simulations of heat transfer in TaylorCouette flow, J. Heat Transfer 120, 65-71 (1998).

[7] C. Vives, Effects of a forced Couette flow during the controlled solidification of a pure metal, Int. J. Heat. Mass Transfer 31, 2047-2062 (1988).

[8] G.B. McFadden, S.R. Coriell and B.T. Murray, Effect of a crystal-melt interface on Taylor-vortex flow, Phys. Fluids A2 (5), 700-705(1990).

[9] R. MacAndrew, N. Parcy, J-M Prieur, J. Wiggelman, E. Diggins, P. Guicheney, D. Cameron and A. Stewart, Drilling and Testing hot, high-pressure wells, Oilfield Review 5, 1532 (1993).

[10] S. Chandrasekhar, Hydrodynamic and Hydromagnetic Stability, Oxford University Press (1961).

[11] P.G. Drazin and W.H. Reid, Hydrodynamic Stability, Cambridge University Press (1981).

[12] G. de Vahl Davis and R.W. Thomas, Natural Convection between concentric vertical cylinders, The Phys. Fluids Supplement II, 198-207 (1969). 
[13] I.G. Choi and S.A. Korpela, Stability of a conduction regime of natural convection in a tall vertical annulus, J. Fluid Mech. 99, 725-738 (1980).

[14] P. Le Quéré and J. Pécheux, Numerical simulation of multiple flow transitions in axisymmetric annulus convection, J. Fluid Mech. 206, 517- 544 (1989).

[15] A. Bahloul, I. Mutabazi and A. Ambari, Codimension 2 points in the flow inside a cylindrical annulus with a radial temperature gradient, Eur. Phys. J. AP 9, 253-264 (2000).

[16] H.A. Snyder and S.K.F. Karlsson, Experiments on the stability of Couette motion with a radial temperature gradient, Phys. Fluids 7, 1696-1706 (1964).

[17] M.M. Sorour and J.E.R. Coney, The effect of temperature gradient on the stability of flow between vertical concentric rotating cylinders, J. Mech. Engng Sci. 21, 403-409 (1979).

[18] K.S. Ball, B. Farouk and V.C. Dixit, An experimental study of heat transfer in a vertical annulus with a rotating inner cylinder, Int. J. Heat Mass Transfer 32, 1517-1527 (1989).

[19] M.E. Ali and P.D. Weidman, On the stability of circular Couette flow motion with radial heating, J. Fluid Mech. 220, 53-84 (1990).

[20] D.C. Kuo and K.S. Ball, Taylor-Couette flow with buoyancy: Onset of spiral flow, Phys. Fluids 9(10), 2872-2884 (1997).

[21] V. Lepiller, A. Goharzadeh, A. Prigent and I. Mutabazi, Weak temperature gradient effect on the stability of circular Couette flow, Eur. J. Phys. B 61, 445-455 (2008).

[22] V. Lepiller, D-H. Yoon, A. Prigent, K-S. Yang and I. Mutabazi, Mixed convection in a differentially rotating annulus, Proceedings of FEDSM2006, 2006 ASME Joint US-European Fluids Engineering Summer Meeting, July 17-20, 2006, Miami, Florida, USA.

[23] H. Yoshikawa, M. Nagata and I. Mutabazi, Instability of the vertical annular flow with a radial heating and rotating inner cylinder, Phys. Fluids 25 (11), 114104 (2013).

[24] C. Kang, K.S.Yang and I. Mutabazi, The effect of radial temperature gradient on the circular-Couette flow,” J. Comput. Fluids Eng. 14(3), 16-24 (2009).

[25] C. Kang, K.S.Yang and I. Mutabazi, Thermal effect on large-aspect-ratio Couette-Taylor system: numerical simulations, J. Fluid Mech.771, 57-78 (2015). 
[26] M. Auer, F.H. Busse and R.M. Clever, Three-dimensional convection driven by centrifugal buoyancy, J. Fluid Mech. 301, 371-382 (1995).

[27] B. Eckhardt, S. Grossmann and D. Lohse, Torque scaling in turbulent Taylor-Couette flow between independently rotating cylinders, J. Fluid Mech. 581, 221-250 (2007).

[28] J. Kim and P. Moin, “Application of a fractional-step method to incompressible NavierStokes equation,” J. Comput. Phys. 59, 308-323 (1985).

[29] J. Jeong and F. Hussain, on the identification of a vortex, J. Fluid Mech. 285, 69-94 (1995).

[30] V. Lepiller, A. Prigent, F. Dumouchel and I. Mutabazi, Transition to turbulence in a tall annulus submitted to a radial temperature gradient, Phys. Fluids 19, 054101 (2007).

[31] P. Matisse and M. Gorman, Neutrally buoyant anisotropic particles for flow visualization, Phys. Fluids 27, 759-760 (1984).

[32] N. Abcha, N. Latrache, F. Dumouchel and I. Mutabazi, Qualitative relation between reflected light intensity by Kalliroscope flakes and velocity field in the Couette-Taylor flow system, Exp. Fluids 45, 85-94 (2008).

[33] C. Savaro, Etude expérimentale de l'effet d'un gradient radial de température sur les instabilités du système de Couette-Taylor, Thèse de doctorat de l'Université du Havre (2014).

[34] R.F. Belgholz, Instability of steady natural convection in a vertical fluid layer, J. Fluid Mech. 84, 743-768 (1978).

[35] P.D. Weidman and G. Mehrdadtehranfar, Instability of natural convection in a tall annulus, Phys. Fluids 28, 776-787 (1985).

[36] M. Ali and P. Weidman, Visualization of the stability of viscous flow between rotating cylinders with a radiall thermal gradient, AAAS Southwestern and Rocky Mountain Division, 62 ${ }^{\text {nd }}$ Annual Meeting, Boulder, CO, 1986.

[37] R. Guillerm, Etude expérimentale des instabilités thermo-hydrodynamiques dans un système de Couette-Taylor, Thèse de doctorat de l'Université du Havre (2010).

[38] B. Martinez Arias, J. Peixinho, O. Crumeyrolle and I. Mutabazi, Effect of the number of vortices on the torque scaling in Taylor-Couette flow, J. Fluid Mech. 748, 756-767 (2014). 
[39] H. Aoki, H. Nohira and H. Arai, Convective heat transfer in an annulus with an inner rotating cylinder, Bulletin of JSME 10, 523-532(1967).

[40] S. Viazzo and S. Poncet, Numerical simulation of the flow stability in a high aspect ratio Taylor-Couette system submitted to a radial temperature gradient, Computers \& Fluids 101, $15-26$ (2014). 